\title{
Supersymmetric perturbations of the M5 brane
}

\author{
Vasilis Niarchos \\ Crete Center for Theoretical Physics \\ $\&$ Crete Center for Quantum Complexity and Nanotechnology, \\ Department of Physics, University of Crete, 71303, Greece \\ E-mail: niarchos@physics.uoc.gr
}

ABSTRACT: We study long-wavelength supersymmetric deformations of brane solutions in supergravity using an extension of previous ideas within the general scheme of the blackfold approach. As a concrete example, we consider long-wavelength perturbations of the planar M2-M5 bound state solution in eleven-dimensional supergravity. We propose a specific ansatz for the first order deformation of the supergravity fields and explore how this deformation perturbs the Killing spinor equations. We find that a special part of these equations gives a projection equation on the Killing spinors that has the same structure as the $\kappa$-symmetry condition of the abelian M5 brane theory. Requiring a match between supergravity and gauge theory implies a specific non-linear gauge-gravity map between the bosonic fields of the abelian M5 brane theory and the gravity-induced fluid-like degrees of freedom of the blackfold equations that control the perturbative gravity solution. This observation sheds new light on the SUGRA/DBI correspondence.

KEYWORDS: p-branes, Gauge-gravity correspondence, Supergravity Models, M-Theory

ArXiv EPRINT: 1402.4132 


\section{Contents}

1 Introduction $\quad 1$

1.1 The forest 1

1.2 The tree 4

2 Abelian M5 brane theory 5

$\begin{array}{lll}2.1 & 1 / 2 \text {-BPS planar solution with uniform } \mathcal{H} \text {-flux } & 6\end{array}$

$\begin{array}{lll}3 & \text { M5 branes in supergravity } & 7\end{array}$

$\begin{array}{lll}3.1 & \text { Supergravity conventions, equations of motion, Killing spinor equations } & 7\end{array}$

3.2 Planar M2-M5 bound state solution 8

3.3 The Killing spinor equations of the planar M2-M5 bound state 9

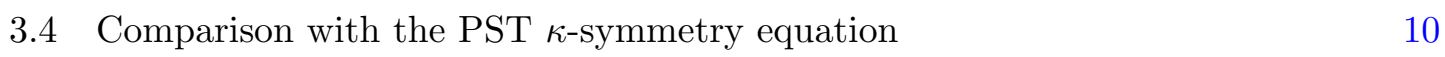

4 Ansatz for extremal M2-M5 deformations 11

$\begin{array}{lll}4.1 & \text { Effective degrees of freedom } & 12\end{array}$

$\begin{array}{lll}4.2 & \text { Near-zone supergravity deformation } & 12\end{array}$

$\begin{array}{lll}4.3 & \text { Bosonic blackfold equations } & 13\end{array}$

5 Killing spinor equations: intrinsic deformations of M2-M5 15

$\begin{array}{lll}5.1 \kappa \text {-symmetry condition for intrinsic perturbations } & 16\end{array}$

6 Killing spinor equations: extrinsic deformations of M2-M5 19

$\begin{array}{lll}6.1 & \kappa \text {-symmetry condition for extrinsic perturbations } & 21\end{array}$

$\begin{array}{lll}7 & \text { Open issues and outlook } & 23\end{array}$

A Summary of perturbative Killing spinor equations $\quad 24$

A.1 Intrinsic perturbations 24

$\begin{array}{lll}\text { A.2 } & \text { Extrinsic perturbations } & 26\end{array}$

B Useful identities $\quad 28$

$\begin{array}{lll}\text { B.1 Consistency check of (5.23) } & 28\end{array}$

B.2 An identity for Killing spinors on $S^{4} \quad 29$

\section{Introduction}

\subsection{The forest}

D-/M-branes in string/M-theory have a rich space of supersymmetric configurations. This space is parameterized by a set of discrete parameters (abstractly, the number $N$ of branes), 
a set of continuous parameters (the moduli), which are vacuum expectation values of gaugeinvariant operators, and the field profiles that define the bulk (closed string) background on which the brane propagates. The expectation values of the moduli may or may not break the underlying gauge symmetry of the brane. For concreteness, we will focus on the subspace of supersymmetric (bosonic) configurations that do not break the gauge symmetry (in a standard system like the system of $N$ D3 branes this would be the origin of the Coulomb branch).

Tracing the properties of this space across $N$ requires a detailed understanding of the theory that resides on the brane. For D-branes in string theory a configuration of the brane (supersymmetric or not) is determined by an exact solution of an open string (field) theory, which, in general, is hard to obtain directly. The common approach to this problem is to identify first an exact solution (a special point $P$ in configuration space) and work in its vicinity by setting up an effective field theory description of long-wavength deformations. $P$ is usually a point with enhanced symmetry where the full open string equations of motion can be solved exactly. The general assumption in all practical applications of this strategy is that solutions of the leading order effective field theory are approximate representations of an exact configuration a finite distance away from $P$.

It is best known how to implement this stategy in the abelian case of a single D-brane in string theory. In a standard derivation (see [1] for a review) $P$ is a brane configuration with a flat worldvolume and a constant gauge field strength $F_{2}$. The resulting effective field theory, which is the well-known abelian Dirac-Born-Infeld (DBI) theory, is determined from a computation of the disk worldsheet partition function or a computation of an infinite set of tree-level scattering amplitudes. The extension to curved backgrounds and the inclusion of general couplings with the background fields is known. However, the understanding of the non-abelian extension of this theory $(N \geq 2)$ is more rudimentary (we refer the reader to the review [2] and references therein).

Configurations that preserve some amount of supersymmetry are special and obey additional conditions. These conditions are nicely packaged in a single projection equation

$$
\boldsymbol{\Gamma}_{\kappa} \epsilon=\epsilon, \quad \boldsymbol{\Gamma}_{\kappa}^{2}=\mathbf{1}
$$

that involves the spinorial supersymmetry transformation parameter $\epsilon[3] . \boldsymbol{\Gamma}_{\kappa}$ is the matrix that controls the $\kappa$-symmetry transformations of the brane theory. The specifics of this matrix depend on the profile of the brane and background fields. The solutions of this equation involve $(i)$ a set of first order differential constraints on the bosonic fields of the brane effective theory, and (ii) a set of differential and algebraic constraints on $\epsilon$ that determine the amount of the preserved supersymmetry.

It is of interest to understand how these structures evolve as we increase the number of branes $N$. Parts of the configuration space can often be traced from the one extreme at $N=1$ to the other at $N \rightarrow \infty$. For instance, configurations at finite $N$ that involve only the abelian (diagonal U(1), center-of-mass) degrees of freedom are always solutions of the same abelian effective DBI theory (up to overall constants). The $N \rightarrow \infty$ regime is particularly interesting, because it is the regime where configurations are described by classical solutions of the bulk supergravity theory. There are many instances in the literature 
where a direct correspondence is observed between solutions of the abelian DBI theory and brane solutions of the bulk supergravity equations of motion (a SUGRA/DBI correspondence). An indicative list of examples includes [4-6]. Connections between $\kappa$-symmetry and calibrations in brane theory and supergravity are also relevant for this aspect (for recent related work see [7]; for an instructive review see [8]). To the best of our knowledge, there is currently no systematic general understanding of this correspondence and part of the motivation of the present work is to provide one.

Such comparisons between the low- $N$ and large- $N$ descriptions require a continuation of the DBI philosophy to large- $N$, namely the notion of a theory of long-wavelength deformations of brane solutions in supergravity. In recent years it has been proposed $[9,10]$ that such a theory can be set up within a scheme of matched asymptotic expansions (see [11] for a detailed discussion of such expansions in the context of caged black holes). Once the zeroth order solution is identified, the constant parameters that control it (e.g. charge densities, rotation parameters, etc.) are promoted to slowly-varying fields of the worldvolume coordinates and the gravity equations are solved perturbatively in a derivative expansion scheme. In the process one discovers that the slowly-varying fields are degrees of freedom of a gravity-induced effective worldvolume theory (coined blackfold theory [9]) that involves a fluid on a dynamical hypersurface. The hypersurface acts in many ways as a holographic screen; it is naturally located in a region far from the black hole horizon and the theory on it is conjectured to control the perturbative solution in the bulk.

This approach can be employed in diverse contexts, e.g. zero or finite temperature, neutral or charged branes, asymptotically flat or asymptotically non-flat backgrounds. For finite-temperature AdS black branes the same process leads naturally to the fluid-gravity correspondence [12], where one recovers an effective fluid on a surface with fixed geometry. The latter is related holographically with the fluid description of a dual strongly coupled quantum field theory. Until now the blackfold approach has been applied successfully in gravity to provide evidence for new black holes solutions with exotic horizon geometries [13-18], in the AdS/CFT correspondence [19, 20], in string/M-theory [21-28]. A recent discussion about the relation of blackfolds, the fluid-gravity correspondence and the membrane paradigm appeared in [29]. A different interesting direction has been pursued in $[30,31]$.

For asymptotically flat neutral black brane solutions in Einstein gravity refs. [32, 33] have shown that the leading order hydrodynamical equations of blackfold theory guarantee the existence of a regular first order corrected solution of the Einstein equations. Regularity refers here to the solution outside the black hole horizon. This result has been partially extended to electrically charged black brane solutions of Einstein-Maxwell gravity in [34]. The generic brane configuration in theories with bulk gauge fields (e.g. in supergravity) involves complex anisotropic fluids with multiple conserved higher-differential-form charge currents. Such descriptions are ubiquitous in the discussion of brane bound states. The elastic and fluid-dynamical properties of these systems are less understood. More importantly, the derivation of the first order corrected supergravity solutions in these more general cases remains a largely open problem.

Supersymmetric solutions provide a fruitful arena for this problem. As a first step in this direction we consider the formulation of supersymmetric perturbations of brane bound 
states within the blackfold expansion scheme. We propose a specific ansatz for the first order deformation of the supergravity fields and demonstrate that a special part of the supergravity Killing spinor equations gives rise to a projection equation that has the same structure as the $\kappa$-symmetry equation (1.1) of the non- gravitational abelian DBI theory. This observation suggests a concrete gauge-gravity map between the degrees of freedom of the DBI description and degrees of freedom derived directly from gravity. As a result, this map adds a new element to the SUGRA/DBI correspondence and opens the road to a deeper understanding of the nature and structure of the dynamical equations of the blackfold effective theory.

As a final comment, we note that there is an old proposal (first applied to string theory in [35]) that identifies the abelian part of the D-/M-brane degrees of freedom (transverse scalars, gauge fields) in supergravity as collective coordinates associated to large gauge transformations. Although this approach shares some qualitative similarities with the blackfold description, the two are significantly different. For instance, the old approach of [35] (for a review see [3]) identifies from supergravity worldvolume abelian gauge fields. In contrast, we will see that the blackfold approach identifies naturally, with a particular nonlinear rewriting that we analyze in this paper, worldvolume abelian gauge field strengths. ${ }^{1}$ Moreover, the blackfold effective theory encodes in a rather straightforward manner the full non-linear nature of the DBI action. This is hard to achieve with the techniques of [35].

\subsection{The tree}

For concreteness, in this paper we will investigate the above construction in the context of a very specific brane configuration in the eleven-dimensional supergravity description of M-theory. Following closely the logic of the string theory derivation of the DBI action [1] we begin with the configuration of an M5 brane in the presence of a constant worldvolume three-form flux. The specifics of this solution and the details of the abelian M5 brane theory proposed in [37-39] are summarized in section 2. In section 3 we move to the supergravity regime and recall the details of a corresponding exactly known supergravity solution that represents a planar M2-M5 bound state. Both gauge theory and gravity solutions are 1/2BPS and the analysis of the Killing spinor equations in supergravity in this simple uniform case reveals immediately a natural connection with the $\kappa$-symmetry equation of the abelian M5 brane theory. Part of this connection is a specific map between the uniform three-form flux of the abelian M5 brane theory and a parameter that controls the M2 brane charge in the supergravity solution.

An ansatz for a general extremal long-wavelength deformation of the seed planar M2M5 solution in supergravity is proposed in section 4. The proposal extends the treatment of neutral black branes in $[32,33]$ to a setting of an extremal two-charge black brane solution.

\footnotetext{
${ }^{1}$ For brane configurations in supergravity interpolating between the asymptotic Minkowski space and a near-horizon AdS space, the abelian nature of the brane effective theory suggests that this theory is a supersingleton field theory. This fact was recognized early on in [36]. The new element that we add to this story is the proposal that this supersingleton field theory is blackfold theory. It would be interesting to explore this connection further.
} 
We summarize the basic assumptions and salient features of the approach and list the relevant bosonic blackfold equations.

The insertion of this ansatz to the supergravity Killing spinor equations gives a lengthy set of complicated equations. In sections 5,6 , which contain the main results of the paper, we notice that there are drastic simplifications if we focus on a specific part of the equations, and that this part has the same structure as the $\kappa$-symmetry equations of the abelian M5 brane theory. Having considered a general inhomogeneous configuration of the brane solution, the correspondence between the gravity-induced and gauge theory $\kappa$-symmetry conditions implies a specific map between the blackfold effective degrees of freedom (which control the form of the supergravity solution) and the abelian M5 brane theory degrees of freedom. This map is a central result of the paper.

We conclude with a summary of the most pressing open issues and an outlook of the approach. Useful technical details are collected in two appendices.

\section{Abelian M5 brane theory}

The theory of a single M5 brane is a six-dimensional abelian theory of the tensor multiplet with sixteen supersymmetries. The latter comprises of two complex Weyl spinors, a selfdual two-form potential $B_{2}$, and five scalar fields. The dynamics of the long-wavelength fluctuations of the brane is controlled by a non-linear effective action of these fields, which is the M5 brane analog of the Dirac-Born-Infeld action for D-branes. Following [38] the bosonic part of this action (in short PST action) reads ${ }^{2}$

$$
\begin{aligned}
S_{M 5}= & -T_{M 5} \int d^{6} \sigma\left(\sqrt{-\operatorname{det}\left(\gamma_{a b}+\tilde{H}_{a b}\right)}+\frac{1}{4} \sqrt{-\operatorname{det} \gamma} \tilde{H}^{a b} \mathcal{H}_{a b c} \mathbf{v}^{c}\right) \\
& +T_{M 5} \int\left(C_{6}+\frac{1}{2} \mathcal{H}_{3} \wedge C_{3}\right) .
\end{aligned}
$$

$T_{M 5}=\frac{1}{(2 \pi)^{5} \ell_{P}^{6}}$ is the M5 brane tension. The scalar fields $X^{\mu}$ (of which only 5 are physical) define the induced worldvolume metric

$$
\gamma_{a b}=g_{\mu \nu} \partial_{a} X^{\mu} \partial_{b} X^{\nu}
$$

where $a, b, \ldots$ are worldvolume indices and $g_{\mu \nu}$ the background metric. The $\mathcal{H}_{3}$ field strength is the gauge invariant 3 -form

$$
\mathcal{H}_{3}=d B_{2}-C_{3} .
$$

$C_{3}$ is the background supergravity 3 -form potential and $C_{6}$ its Hodge dual. The self-duality of the worldvolume 2-form potential is expressed nicely in the PST formulation with the use of an auxiliary scalar field $\varphi$ (usually referred to as $a$ in the literature). The derivatives of this field define the unit vector

$$
\mathbf{v}_{a}=\frac{\partial_{a} \varphi}{\sqrt{-\partial_{b} \varphi \partial^{b} \varphi}}
$$

\footnotetext{
${ }^{2}$ See also [40] for a recent reformulation of the M5 brane action in a ' $3+3$ ' split.
} 
which appears in (2.1). In (2.1) we are also using the definitions

$$
\tilde{H}_{a b}=\mathcal{H}_{a b c}^{*} \mathbf{v}^{c}, \quad \mathcal{H}^{* a b c}=\frac{1}{3 !} \frac{1}{\sqrt{-\operatorname{det} \gamma}} \varepsilon^{a b c d_{1} d_{2} d_{3}} \mathcal{H}_{d_{1} d_{2} d_{3}} .
$$

Notice that the physical bosonic degrees of freedom of the tensor multiplet in six dimensions are 8: 5 from the scalars and 3 from the self-dual 2-form. The PST action is expressed in terms of $X^{\mu}, \mathbf{v}_{a}, \tilde{H}_{a b}$, which have in total 25 components. ${ }^{3}$ In the supergravity analysis below we will also encounter a 25-component set of bosonic fields arising directly from the gravitational degrees of freedom.

The supersymmetric bosonic configurations of the M5 brane (which will be the main focus of this paper) obey the projection equation

$$
\boldsymbol{\Gamma}_{\kappa} \epsilon=\epsilon
$$

where $\epsilon$ is a Majorana spinor in eleven-dimensions and $\boldsymbol{\Gamma}_{\kappa}$ the M5 brane $\kappa$-symmetry matrix

$$
\boldsymbol{\Gamma}_{\kappa}=\frac{\mathbf{v}_{a} t_{b}}{\sqrt{-\operatorname{det}(\gamma+\tilde{H})}} \Gamma^{a b}+\frac{\sqrt{-\operatorname{det} \gamma} \mathbf{v}_{a} \tilde{H}_{b c}}{2 \sqrt{-\operatorname{det}(\gamma+\tilde{H})}} \Gamma^{a b c}-\frac{\varepsilon_{a_{1} \ldots a_{5} b} \mathbf{v}^{b} \mathbf{v}_{a}}{5 ! \sqrt{-\operatorname{det}(\gamma+\tilde{H})}} \Gamma^{a} \Gamma^{a_{1} \ldots a_{5}} .
$$

We defined

$$
t^{a}=\frac{1}{8} \varepsilon^{a b_{1} b_{2} c_{1} c_{2} d} \tilde{H}_{b_{1} b_{2}} \tilde{H}_{c_{1} c_{2}} \mathbf{v}_{d}
$$

and $\Gamma^{a}$ are curved worldvolume $\Gamma$-matrices.

\section{$2.1 \quad 1 / 2-B P S$ planar solution with uniform $\mathcal{H}$-flux}

Before ending this section we summarize the properties of a $1 / 2$-BPS solution that will soon play a protagonistic role in our discussion.

A simple solution of the PST equations of motion in flat space with trivial planar worldvolume geometry has (in the temporal gauge)

$$
\mathbf{v}^{a}=(1,0,0,0,0,0)
$$

and constant $\mathcal{H}$-flux with non-vanishing components ${ }^{4}$

$$
\mathcal{H}_{012}=\frac{H}{\sqrt{1+H^{2}}}, \quad \mathcal{H}_{345}=H, \quad H=\text { constant } .
$$

For this profile $\tilde{H}_{12}=H$, the vector $t$ is vanishing and the $\kappa$-symmetry matrix is

$$
\Gamma_{\kappa}=-\frac{1}{\sqrt{1+H^{2}}}\left(H \Gamma_{\|}+\Gamma_{\|} \Gamma_{\perp}\right)
$$

\footnotetext{
${ }^{3} X^{\mu}$ has 11 components, $\mathbf{v}_{a}$ as a unit worldvolume vector has 5 , and $\tilde{H}_{a b}$ has $15-6=9$ because of anti-symmetry and the defining relation $\mathbf{v}^{a} \tilde{H}_{a b}=0$. Several gauge invariances operate on this system. For example, one can use a symmetry that shifts the auxiliary field $\varphi$ (and at the same time transforms the 2 -form potential $B_{2}$ ) to fix the vector $\mathbf{v}_{a}$ and obtain a non-manifest Lorentz invariant formulation of the M5 brane effective theory [41].

${ }^{4}$ For a general parametrization of constant $\mathcal{H}$-flux solutions see [42]. Such solutions give rise to a noncommutative M5 brane theory and play a role in the general setup of OM theory [43].
} 
where $\Gamma_{\|}=\Gamma^{0} \Gamma^{1} \Gamma^{2}$ and $\Gamma_{\perp}=\Gamma^{3} \Gamma^{4} \Gamma^{5}$. Defining an angle $\theta$ such that ${ }^{5}$

$$
\cos \theta=\frac{1}{\sqrt{1+H^{2}}}, \quad \sin \theta=\frac{H}{\sqrt{1+H^{2}}}
$$

the $\kappa$-symmetry equation (2.6) becomes

$$
\left(1+\sin \theta \Gamma_{\|}+\cos \theta \Gamma_{\|} \Gamma_{\perp}\right) \epsilon=0 .
$$

This equation implies the reduction of the original thirty-two supersymmetries of the background by one half.

Since the $\mathcal{H}$-flux induces M2 brane charge this solution is naturally interpreted as a 1/2-BPS state of the M5 with M2 brane charge along the directions (012) uniformly smeared along the transverse (345) plane inside the M5 brane worldvolume.

\section{$3 \quad$ M5 branes in supergravity}

We now turn to the opposite regime where an infinite number of overlapping M5 branes is described by an extremal solution of the eleven-dimensional supergravity. In this section we fix our notation and present the symmetric solution whose long-wavelength deformations we will study later.

\subsection{Supergravity conventions, equations of motion, Killing spinor equations}

We shall use small Greek letters $\mu, \nu, \ldots$ to denote the curved spacetime indices and hatted small Greek letters $\hat{\mu}, \hat{\nu}, \ldots$ to denote tangent flat spacetime indices. Small latin letters $a, b, \ldots$ will be employed for spacetime directions parallel to the M5 brane worldvolume. The components of the metric, vielbein and spin connection are denoted respectively as $g_{\mu \nu}, e_{\mu}^{\hat{\nu}}, \omega_{\mu}^{\hat{\nu} \hat{\rho}}$. Hodge duals in the eleven-dimensional spacetime will be written using a $\star$, and Hodge duals on effective worldvolumes later using a $*$.

The bosonic part of the eleven-dimensional supergravity action is

$$
I_{\text {sugra }}=\frac{1}{2 \kappa_{11}^{2}} \int d^{11} x \sqrt{-g}\left(R-\frac{1}{2 \cdot 4 !} F_{\mu_{1} \mu_{2} \mu_{3} \mu_{4}} F^{\mu_{1} \mu_{2} \mu_{3} \mu_{4}}\right)-\frac{1}{12 \kappa_{11}^{2}} \int C_{3} \wedge F_{4} \wedge F_{4}
$$

where $\kappa_{11}$ is the eleven-dimensional Newton constant and $F_{4}=d C_{3}$ the four-form field strength. The equations of motion for the metric and gauge field are

$$
\begin{aligned}
R_{\mu \nu}-\frac{1}{12}\left(\left(F_{4}^{2}\right)_{\mu \nu}-\frac{1}{12} g_{\mu \nu} F_{4}^{2}\right) & =0 \\
d \star F_{4}+\frac{1}{2} F_{4} & \wedge F_{4}=0
\end{aligned}
$$

where the shorthand notation $F_{4}^{2}=F_{\mu_{1} \mu_{2} \mu_{3} \mu_{4}} F^{\mu_{1} \mu_{2} \mu_{3} \mu_{4}}$ and $\left(F_{4}^{2}\right)_{\mu \nu}=F_{\mu \rho_{1} \rho_{2} \rho_{3}} F_{\nu}^{\rho_{1} \rho_{2} \rho_{3}}$ was employed. In addition we have the Bianchi identity

$$
d F_{4}=0
$$

\footnotetext{
${ }^{5}$ In this definition $\theta \in\left[-\frac{\pi}{2}, \frac{\pi}{2}\right]$. With a parity transformation $\theta$ extends over the full range $[0,2 \pi)$.
} 
The supergravity multiplet also includes a single spin-3/2 field, the gravitino $\psi$. Bosonic supersymmetric configurations with $\psi=0$ require the presence of a residual supersymmetry expressed in terms of a Majorana spinor $\eta$ that obeys the Killing spinor equation

$$
\nabla_{\mu} \eta+\frac{1}{288}\left(\Gamma_{\mu}^{\nu_{1} \nu_{2} \nu_{3} \nu_{4}}-8 \delta_{\mu}^{\nu_{1}} \Gamma^{\nu_{2} \nu_{3} \nu_{4}}\right) F_{\nu_{1} \nu_{2} \nu_{3} \nu_{4}} \eta=0
$$

We are using the standard notation where $\Gamma^{\mu \nu \ldots}$ denotes the antisymmetrized product of $\Gamma$-matrices $\Gamma^{\mu}=e_{\hat{\nu}}^{\mu} \Gamma^{\hat{\nu}}$ obeying the Clifford algebra $\left\{\Gamma^{\mu}, \Gamma^{\nu}\right\}=2 g^{\mu \nu}$.

It is known [44] that by using the Killing spinor equation (3.5) on the identity

$$
\nabla_{[\rho} \nabla_{\mu]} \eta=\frac{1}{8} R_{\rho \mu \sigma_{1} \sigma_{2}} \Gamma^{\sigma_{1} \sigma_{2}} \eta
$$

one can deduce the equation

$$
\begin{aligned}
0= & {\left[R_{\rho \mu}-\frac{1}{12}\left(\left(F_{4}^{2}\right)_{\rho \mu}-\frac{1}{12} g_{\rho \mu} F_{4}^{2}\right)\right] \Gamma^{\mu} \eta } \\
& -\frac{1}{6 \cdot 3 !} \star\left(d \star F_{4}+\frac{1}{2} F_{4} \wedge F_{4}\right)_{\sigma_{1} \sigma_{2} \sigma_{3}}\left(\Gamma_{\rho}^{\sigma_{1} \sigma_{2} \sigma_{3}}-6 \delta_{\rho}^{\sigma_{1}} \Gamma^{\sigma_{2} \sigma_{3}}\right) \eta \\
& -\frac{1}{6 !}\left(d F_{4}\right)_{\sigma_{1} \sigma_{2} \sigma_{3} \sigma_{4} \sigma_{5}}\left(\Gamma_{\rho}^{\sigma_{1} \sigma_{2} \sigma_{3} \sigma_{4} \sigma_{5}}-10 \delta_{\rho}^{\sigma_{1}} \Gamma^{\sigma_{2} \sigma_{3} \sigma_{4} \sigma_{5}}\right) \eta,
\end{aligned}
$$

which implies, for example, that the Einstein equations follow automatically from the combination of the Killing spinor equations, the Bianchi identity and the equations of motion of $F_{4}$. This observation will be useful in the next section.

\subsection{Planar M2-M5 bound state solution}

The starting point of our discussion in the next section is an exact solution of the supergravity equations (3.2)-(3.4) that describes a planar 1/2-BPS M2-M5 bound state [45] (see also $[46,47])$

$$
\begin{aligned}
& d s^{2}=(H D)^{-\frac{1}{3}}[-\left(d x^{0}\right)^{2}+\left(d x^{1}\right)^{2}+\left(d x^{2}\right)^{2}+D\left(\left(d x^{3}\right)^{2}+\left(d x^{4}\right)^{2}+\left(d x^{5}\right)^{2}\right) \\
&\left.+H\left(d r^{2}+r^{2} d \Omega_{4}^{2}\right)\right] \\
& F_{4}=d \mathcal{C}_{3}+D^{-1} \star d \mathcal{C}_{6}
\end{aligned}
$$

where

$$
\begin{aligned}
& \mathcal{C}_{3}=-\sin \theta\left(H^{-1}-1\right) d x^{0} \wedge d x^{1} \wedge d x^{2}+\tan \theta D H^{-1} d x^{3} \wedge d x^{4} \wedge d x^{5}, \\
& \mathcal{C}_{6}=\cos \theta D\left(H^{-1}-1\right) d x^{0} \wedge d x^{1} \wedge d x^{2} \wedge d x^{3} \wedge d x^{4} \wedge d x^{5}, \\
& H=1+\frac{r_{H}^{3}}{r^{3}}, \quad D^{-1}=\cos ^{2} \theta+\sin ^{2} \theta H^{-1} .
\end{aligned}
$$

The solution is parametrized by two constants, $\theta$ and $r_{H}$, that control the energy density $\varepsilon$ and the M2 and M5 charge densities $\left(\mathcal{Q}_{2}, \mathcal{Q}_{5}\right.$ respectively)

$$
\varepsilon=\frac{\Omega_{(4)}}{16 \pi G} r_{H}^{3}, \quad \mathcal{Q}_{2}=-\sin \theta Q, \quad \mathcal{Q}_{5}=\cos \theta Q, \quad Q=\frac{3 \Omega_{(4)}}{16 \pi G}, \quad 8 \pi G=\kappa_{11}^{2} .
$$

$\Omega_{(4)}=8 \pi^{2} / 3$ is the volume of the unit round 4 -sphere. 
The uniformly smeared M2-brane charge along the transverse (345) plane breaks the isotropy of the M5 brane worldvolume plane (012345) in direct correspondence with the breaking that was noted in the non-gravitational constant $\mathcal{H}$-flux solution of a single M5 brane (2.9)-(2.10) in section 2.1.

\subsection{The Killing spinor equations of the planar M2-M5 bound state}

As warmup for the more complicated analysis that follows it is instructive to recall how the profile (3.9)-(3.13) solves the Killing spinor equations (3.5).

The spacetime coordinates split naturally into the four groups: $\left(x^{0}, x^{1}, x^{2}\right),\left(x^{3}, x^{4}, x^{5}\right)$, $r$ and $\left(y^{1}, y^{2}, y^{3}, y^{4}\right)$ for the transverse $S^{4}$. Accordingly, we define the following antisymmetric combinations of the flat space $\Gamma$-matrices

$$
\Gamma_{\|}=\Gamma^{\hat{0} \hat{1} \hat{2}}, \quad \Gamma_{\perp}=\Gamma^{\hat{3} \hat{4} \hat{5}}, \quad \Gamma_{\Omega}=\Gamma^{\hat{y}^{1} \hat{y}^{2} \hat{y}^{3} \hat{y}^{4}} .
$$

These combinations together with $\Gamma_{\hat{r}}$ obey the identity

$$
\Gamma_{\|} \Gamma_{\perp} \Gamma_{\Omega} \Gamma_{\hat{r}}=1
$$

For notational economy it is also convenient to define the functions

$$
\begin{aligned}
& e^{S}=H D, \quad e^{Q}=H D^{-2}, \quad e^{R}=r^{-6} H^{-2} D, \\
& f_{1}=-\sin \theta\left(H^{-1}-1\right), \quad f_{2}=\tan \theta D H^{-1}, \quad f_{3}=\cos \theta D\left(H^{-1}-1\right) \text {, }
\end{aligned}
$$

which are all functions of the radial coordinate $r$.

With these specifications the covariant derivatives of the spinor $\eta$ are

$$
\begin{aligned}
\nabla_{\mu} \eta & =\partial_{\mu} \eta+\frac{1}{12} \partial^{\nu} S \Gamma_{\nu \mu} \eta, & \mu & =0,1,2, \\
\nabla_{\mu} \eta & =\partial_{\mu} \eta+\frac{1}{12} \partial^{\nu} Q \Gamma_{\nu \mu} \eta, & \mu & =3,4,5, \\
\nabla_{y^{i}} \eta & =\tilde{\nabla}_{y^{i}} \eta+\frac{1}{12} \partial^{\nu} R \Gamma_{\nu y^{i}} \eta, & i & =1,2,3,4,
\end{aligned}
$$

where $\tilde{\nabla}$ is the covariant derivative on the unit $S^{4}$. In the background (3.9)-(3.13) we take $\eta$ to be an $r$-dependent Killing spinor on $S^{4}$ [48], namely we require

$$
\partial_{\mu} \eta=0 \quad(\mu=0,1, \ldots, 5), \quad \tilde{\nabla}_{y^{i}} \eta=\frac{C}{2} e^{\frac{R}{6}} \Gamma_{\Omega} \Gamma_{y^{i}} \eta, \quad C= \pm 1 .
$$

The Killing spinor equation (3.5) can now be recast into the form

$$
\nabla_{\mu} \eta+\frac{1}{288}\left(-\frac{1}{2} \Gamma_{\mu} \not H+\frac{3}{2} \not F \Gamma_{\mu}\right) \eta=0
$$

where

$$
\begin{aligned}
\frac{1}{288} \not H & =F_{\|}+F_{\perp}+F_{\Omega}, \\
F_{\|} & =\frac{1}{12} e^{\frac{S}{2}} \not \partial f_{1} \Gamma_{\|}, \quad F_{\perp}=\frac{1}{12} e^{\frac{Q}{2}} \not \partial f_{2} \Gamma_{\perp}, \quad F_{\Omega}=\frac{1}{12} r^{-2} e^{-\frac{R}{3}} D^{-1} \partial_{r} f_{3} \Gamma_{\Omega} .
\end{aligned}
$$


Writing out each of the components of (3.23) we obtain four equations

$$
\begin{aligned}
\left(-\frac{1}{12} \not \partial S+F_{\perp}+F_{\Omega}-2 F_{\|}\right) \eta & =0, \\
\left(-\frac{1}{12} \not \partial Q+F_{\|}+F_{\Omega}-2 F_{\perp}\right) \eta & =0, \\
\partial_{r} \eta-\Gamma_{r}\left(F_{\|}+F_{\perp}-2 F_{\Omega}\right) \eta & =0, \\
-\frac{C}{2} e^{\frac{R}{6}} \Gamma_{\Omega} \eta+\left(-\frac{1}{12} \not \partial R+F_{\|}+F_{\perp}-2 F_{\Omega}\right) \eta & =0 .
\end{aligned}
$$

By adding equations (3.26), (3.27), (3.29), multiplying on the left by $\Gamma_{r}$, and using (3.16) we obtain the projection equation

$$
\Gamma_{\|} \Gamma_{\perp} \eta=C \eta
$$

Since both values of $C= \pm 1$ are allowed this equation is not restrictive for $\eta$. With a similar manipulation of the linear combination $(3.26)+2 \times(3.27)$ (or equivalently from $2 \times(3.26)$ $+(3.27))$ we obtain a second projection equation

$$
\left(1+D^{\frac{1}{2}} H^{-\frac{1}{2}} \sin \theta \Gamma_{\|}+D^{\frac{1}{2}} \cos \theta \Gamma_{\|} \Gamma_{\perp}\right) \eta=0 .
$$

Finally, (3.28) combined with (3.29) and (3.30) provides the differential equation

$$
\partial_{r} \eta-\frac{1}{12} \partial_{r} \log \left(H^{-2} D\right) \eta=0 .
$$

The general solution of the system (3.31)-(3.32) is expressed in terms of a constant spinor with sixteen independent components [45], verifying the $1 / 2$-BPS nature of the bound state.

\subsection{Comparison with the PST $\kappa$-symmetry equation}

Immediate and intuitive information about the number of preserved supersymmetries is obtained at the asymptotic infinity by analyzing the leading order form of the system (3.31)(3.32) in a $1 / r$-expansion around $r=\infty$. At leading order the spinor $\eta$ is constant, $\eta=\eta_{0}+\mathcal{O}\left(r^{-3}\right)$, equation (3.32) is trivial and the projection equation (3.31) becomes

$$
\left(1+\sin \theta \Gamma_{\|}+\cos \theta \Gamma_{||} \Gamma_{\perp}\right) \eta_{0}=0 .
$$

The interesting, simple-minded observation is that this is the same as the $\kappa$-symmetry equation (2.13) of a single M5 brane with the gauge-gravity identification of fields (2.12).

The main purpose of the ensuing sections is to exhibit how a similar analysis at infinity of more generic M5 brane configurations produces a supergravity-induced $\kappa$-symmetry-type equation and how this compares with the original $\kappa$-symmetry equation of the abelian PST theory. 


\section{Ansatz for extremal M2-M5 deformations}

After a general $\mathrm{SO}(1,5)$ rotation of the M5 brane worldvolume coordinates

$$
\sigma^{a} \rightarrow M_{b}^{a} \sigma^{b}, \quad a=0,1, \ldots, 5, \quad M \in \mathrm{SO}(1,5)
$$

the solution (3.9)-(3.13) takes the form

$$
\begin{aligned}
d s^{2} & =\left(e^{-\frac{S}{3}} \hat{h}_{a b}+e^{-\frac{Q}{3}} \hat{\perp}_{a b}\right) d \sigma^{a} d \sigma^{b}+e^{-\frac{R}{3}}\left(r^{-2} d r^{2}+d \Omega_{4}^{2}\right), \\
\mathcal{C}_{3} & =-\sin \theta\left(H^{-1}-1\right) \omega_{3}-\tan \theta D H^{-1} *_{6} \omega_{3}, \quad \mathcal{C}_{6}=\cos \theta D\left(H^{-1}-1\right) \omega_{6} .
\end{aligned}
$$

We used the $\mathrm{SO}(1,5)$ matrix elements $M_{b}^{a}$ to define three unit orthonormal vectors $u, v, w$ with components

$$
u_{a}=M_{a}^{0}, \quad v_{a}=M_{a}^{1}, \quad w_{a}=M_{a}^{2} .
$$

This identification implies the orthonormality conditions (indices are lowered and raised with the six-dimensional Minkowski metric $\eta_{a b}$ )

$$
u_{a} u^{a}=-1, \quad v_{a} v^{a}=w_{a} w^{a}=1, \quad u_{a} v^{a}=0, \quad u_{a} w^{a}=0, \quad v_{a} w^{a}=0 .
$$

The tensors appearing in (4.2), (4.3) are expressed in terms of these vectors as follows

$$
\begin{aligned}
\hat{h}_{a b} & =-u_{a} u_{b}+v_{a} v_{b}+w_{a} w_{b}, \quad \hat{\perp}_{a b}=\eta_{a b}-\hat{h}_{a b}, \\
\omega_{3} & =u \wedge v \wedge w, \quad \omega_{6}=\sqrt{-\operatorname{det} \eta} d \sigma^{0} \wedge d \sigma^{1} \wedge d \sigma^{2} \wedge d \sigma^{3} \wedge d \sigma^{4} \wedge d \sigma^{5} .
\end{aligned}
$$

The Hodge dual $*_{6}$ is taken with respect to the $6 \mathrm{~d}$ metric $\eta_{a b}$.

The appearance of the vectors $u, v, w$ is a consequence of the breaking of the $\mathrm{SO}(1,5)$ Lorentz symmetry induced by the presence of the smeared M2 brane charge. Accordingly, the tensors $\hat{h}_{a b}$ and $\omega_{3}$ are respectively a projector and a volume three-form along the M2 brane directions, and $\hat{\perp}_{a b}$ is the orthogonal projector.

Our goal is to determine the general supersymmetric deformations of the solution (4.2), (4.3) in a long-wavelength expansion scheme. In the spirit of the fluid-gravity correspondence in AdS [12], or the general blackfold approach [9, 10], such a scheme arises essentially by promoting the parameters that control the zeroth order solution to slowly varying functions of the coordinates $\sigma^{a}$ and appropriately correcting the form of the solution order-by-order in the expansion to satisfy the supergravity equations of motion. The detailed construction of the perturbative solution requires the implementation of a technically complicated matched-asymptotic-expansion scheme where the supergravity equations are solved independently order-by-order in a near-zone and a far-zone region and then matched over a large intermediate region, called the overlap-zone (see [13] for a detailed implementation of this scheme on black rings in neutral, pure Einstein gravity). In the case at hand, deformations with a characteristic long-wavelength scale $\mathcal{R} \gg r_{H}$ define a near-zone that lies radially in the region $r \ll \mathcal{R}$ and a far-zone that lies in the region $r \gg r_{H} \cdot r_{H}$ is the scale appearing in (3.13). The large overlap region lies at distances $r$ such that $r_{H} \ll r \ll \mathcal{R}$. 


\subsection{Effective degrees of freedom}

In this scheme the promoted parameters become naturally the degrees of freedom of an effective six-dimensional worldvolume theory, which is thought to reside in the overlap zone. For deformations of the solution (4.2), (4.3) these parameters are

$$
r_{H}, \quad \theta, u, v, w, X^{\mu} .
$$

The scalars $X^{\mu}$ are degrees of freedom related to the breaking of the trasverse $\mathrm{SO}(5)$ symmetry and parametrize the promotion of the worldvolume metric $\eta_{a b}$ to the general induced metric

$$
\gamma_{a b}=g_{\mu \nu}^{(0)} \partial_{a} X^{\mu} \partial_{b} X^{\nu}
$$

$g_{\mu \nu}^{(0)}$ is the asymptotic value of the bulk metric; here $g_{\mu \nu}^{(0)}=\eta_{\mu \nu}$.

In total, this effective worldvolume description of the gravitational dynamics gives rise to a formulation in terms of 25 degrees of freedom: 2 from $\left(r_{H}, \theta\right)$ that control the M2 and M5 charges, $3 \times 6-3 \times 2=12$ from the unit orthonormal vectors $u, v, w$, and 11 from the scalars $X^{\mu}$. As we noted above (see paragraph after eq. (2.5)), the same number of parameters appears in the abelian PST effective action (2.1) and $\kappa$-symmetry matrix (2.7) (including a vector built out of the auxiliary field $\varphi$ of PST that enforces the self-duality of the 2-form gauge field). Besides the transverse scalars that have an obviously common origin in both the gauge and gravity descriptions, the rest of the degrees of freedom have a dramatically different looking form on each side.

\subsection{Near-zone supergravity deformation}

We proceed to set up a specific ansatz for the first order deformation of the supergravity fields. For the purposes of this paper, we will focus exclusively on the form of this ansatz in the near-zone region. Our proposal is motivated by analogous deformations of black brane solutions in pure Einstein gravity (see $[13,32,33]$ ). It will be shown to be non-trivially consistent with known or expected properties.

Following [33] (suitably extended to include all the fields of eleven-dimensional supergravity) we propose the following first order deformation of the bosonic fields (4.2), (4.3) ( $\boldsymbol{\delta}$ is a dummy variable that keeps track of the deformation order)

$$
\begin{aligned}
d s^{2} & =\left(e^{-\frac{S}{3}} \hat{h}_{a b}+e^{-\frac{Q}{3}} \hat{\perp}_{a b}\right) d \sigma^{a} d \sigma^{b}+e^{-\frac{R}{3}}\left(r^{-2} d r^{2}+d \Omega_{4}^{2}\right)+\boldsymbol{\delta} h_{\mu \nu}(x) d x^{\mu} d x^{\nu}+\mathcal{O}\left(\boldsymbol{\delta}^{2}\right), \\
F_{4} & =d \mathcal{C}_{3}+D^{-1} \star d \mathcal{C}_{6}+\boldsymbol{\delta} G_{4}+\mathcal{O}\left(\boldsymbol{\delta}^{2}\right)
\end{aligned}
$$

with

$$
\mathcal{C}_{3}=-\sin \theta\left(H^{-1}-1\right) \omega_{3}-\tan \theta D H^{-1} *_{6} \omega_{3}, \quad \mathcal{C}_{6}=\cos \theta D\left(H^{-1}-1\right) \omega_{6} .
$$

There are two new elements in these expressions, compared to the seed profile (4.2), (4.3). First, we have promoted all the previously constant parameters (4.8) to $\sigma^{a}$-dependent fields. The functions $S, Q, R$ are still defined in terms of $H, D$ as in (3.17), (3.13), but they 
are now functions of both $r$ and $\sigma^{a}$ since $r_{H}=r_{H}\left(\sigma^{a}\right), \theta=\theta\left(\sigma^{a}\right)$. The vectors $u, v, w$ are also functions of $\sigma^{a}$ on an effective curved worldvolume with induced metric $\gamma_{a b}$ (4.9). The projectors $\hat{h}_{a b}, \hat{\perp}_{a b}$ and the forms $\omega_{3}, \omega_{6}$ are now

$$
\begin{aligned}
\hat{h}_{a b} & =-u_{a} u_{b}+v_{a} v_{b}+w_{a} w_{b}, & \hat{\perp}_{a b} & =\gamma_{a b}-\hat{h}_{a b}, \\
\omega_{3} & =u \wedge v \wedge w, & \omega_{6} & =\sqrt{-\operatorname{det} \gamma} d \sigma^{0} \wedge d \sigma^{1} \wedge d \sigma^{2} \wedge d \sigma^{3} \wedge d \sigma^{4} \wedge d \sigma^{5}
\end{aligned}
$$

and the Hodge dual $*_{6}$ is taken with respect to the $6 \mathrm{~d}$ induced metric $\gamma_{a b}$.

The second modification includes the metric and 4-form corrections $h_{\mu \nu}$ and $G_{4}$. These are of the same order, $\mathcal{O}(\boldsymbol{\delta})$, as the $\sigma$-derivatives of $\log r_{H}, \cos \theta, u, v, w$ and the derivatives of the velocities $\partial_{a} X^{\mu}$. They need to be included in order to satisfy the supergravity equations at first order.

As in ref. [33] the main strategy is to work locally around an arbitrary point on the effective worldvolume. We assume that all the $\sigma^{a}$-dependent fields are slowly-varying fields of the worldvolume coordinates, we expand them in a derivative expansion and work linearly in the perturbations. In this local linearization of the perturbations, the fluctuations split naturally into two decoupled sets that can be analyzed independently.

The first set includes the intrinsic fluctuations, namely fluctuations that are neutral under the 'R-symmetry' generators of the $\mathrm{SO}(5)$ that rotates the five-dimensional space transverse to the brane. These are fluctuations of $r_{H}, \theta, u, v, w$ and the induced metric. The metric fluctuations are subleading to the first order that we will be considering and can be neglected.

The second set includes the extrinsic fluctuations. These are fluctuations of the transverse scalars $X^{\perp}$, which, by definition, are charged under the transverse $\mathrm{SO}(5)$.

The resulting perturbed bosonic fields are then inserted into the supergravity equations of motion (3.2)-(3.4) which are solved perturbatively to determine the deformed solution in the near-zone region.

At the level of supersymmetry the above deformations induce a corresponding perturbative expansion of the Killing spinor equations (3.5). At first order the Majorana spinor $\eta$ is perturbed independently by the intrinsic and extrinsic fluctuations. One of the main goals of this paper is to exhibit the details of this perturbation. The intrinsic perturbative Killing spinor equations will be discussed in section 5 and the extrinsic ones in section 6 .

\subsection{Bosonic blackfold equations}

The implementation of the above scheme on the bosonic supergravity equations (3.2)(3.4) produces a set of partial differential equations involving the effective degrees of freedom (4.8) and the field perturbations $h_{\mu \nu}, G_{4}$ in (4.10)-(4.11). A subset of these equations are constraint equations; they do not involve second derivatives of the radial coordinate, and do not involve the corrections $h_{\mu \nu}, G_{4}$. These equations can be analyzed most easily in the asymptotic infinity of the overlap zone $\left(\mathcal{R} \gg r \gg r_{H}\right)$, where they yield a set of dynamical equations for the effective degrees of freedom (4.8) only, the so-called blackfold equations. 
For the M2-M5 bound state in flat space the leading-order blackfold equations are ${ }^{6}$ (a supergravity derivation of these equations has been performed in [49])

$$
\begin{aligned}
D_{a} T^{a b} & =0, \quad d\left(J_{3}+*_{6} J_{3}\right)=0, \quad d J_{6}=0, \\
K_{a b}{ }^{\rho} T^{a b} & =0
\end{aligned}
$$

where $T^{a b}, J_{3}$ and $J_{6}$ are respectively the stress-energy tensor, M2 brane current, and M5 brane current of the effective worldvolume theory. $K_{a b}{ }^{\rho}$ is the extrinsic curvature tensor for the induced metric $\gamma_{a b}$ (see [10] for detailed expressions) and $D_{a}$ the worldvolume covariant derivative. All these quantities are functionals of the fields (4.8)

$$
\begin{aligned}
T^{a b} & =\tilde{Q} r_{H}^{3}\left(\sin ^{2} \theta \hat{h}^{a b}+\cos ^{2} \theta \gamma^{a b}\right), \\
J_{3} & =-\tilde{Q} \sin \theta r_{H}^{3} \omega_{3}, \quad J_{6}=\tilde{Q} \cos \theta r_{H}^{3} \omega_{6}, \quad \tilde{Q}=\frac{3 \Omega_{(4)}}{4 G} .
\end{aligned}
$$

The first set of equations (4.15) comes from the analysis of the intrinsic fluctuations and the second (4.16) from the extrinsic. More specifically, the first equation in (4.15) and equation (4.16) originate from the analysis of a particular combination of the metric equations (3.2). Similarly, the conservation of the self-dual part of the current $J_{3}$ in (4.15) comes from a component of the gauge-field equation (3.3). The final equation in (4.15) comes from the Bianchi identity (3.4).

We notice that the six-current conservation, $d J_{6}=0$, gives a simple constant of motion

$$
\cos \theta r_{H}^{3}=\text { constant . }
$$

Hence, when inserted into the remaining equations we obtain

$$
\begin{aligned}
D_{a}\left(\frac{\sin ^{2} \theta}{\cos \theta} \hat{h}^{a b}+\cos \theta \gamma^{a b}\right) & =0, \\
\sin ^{2} \theta \hat{h}^{a b} K_{a b}{ }^{\rho}+\cos ^{2} \theta \gamma^{a b} K_{a b}{ }^{\rho} & =0, \\
d\left[\tan \theta\left(\omega_{3}+*_{6} \omega_{3}\right)\right] & =0 .
\end{aligned}
$$

This system of dynamical equations for the 25 effective worldvolume fields of the blackfold expansion should be compared to the equations of motion of the PST action (2.1). Accumulating evidence from explicit solutions of the DBI/PST theory and supergravity (e.g. the BIon [21, 22] and self-dual string soliton solutions [23]) suggests that the extremal equations of the supergravity/blackfold theory are equivalent to the equations of the abelian DBI/PST theory. The precise connection between the two, however, is not immediately obvious at the level of the bosonic equations.

The generic configuration obeying (4.19)-(4.22) is extremal but not supersymmetric. For example, it can be an extremal, time-independent configuration (see [25] for a stationary extremal configuration of the M2-M5-KKW system). In order to determine the

\footnotetext{
${ }^{6}$ This set of equations has already appeared in [23, 25], where it was employed in the analysis of solutions that describe configurations of M2 branes ending orthogonally on M5 branes. In [23, 25] the M2 brane current conservation equation $d\left(J_{3}+*_{6} J_{3}\right)=0$ was mistakenly reported as $d *_{6} J_{3}=0$. However, for the solutions analyzed in $[23,25]$ the missing term $d J_{3}$ is automatically zero and does not affect the results.
} 
supersymmetric subset of solutions we need to analyze the supergravity Killing spinor equations (3.5). Implementing the long-wavelength expansion of section 4.2 on the Killing spinor equations we will soon derive the supergravity analog of the $\kappa$-symmetry equation (2.6). Ultimately, this is expected to allow us to determine which of the extremal blackfold configurations are supersymmetric and how many supersymmetries are preserved by the full supergravity solution.

We note that according to the identity (3.7) the independent subset of dynamical equations for supersymmetric configurations are, e.g., the Killing spinor equations, the equations of motion of the gauge field $C_{3}$ and its Bianchi identity. The statement that appears to emerge out of this identity in the effective blackfold theory is that the set \{supergravityinduced $\kappa$-symmetry-like condition $\oplus$ charge current equations $\}$ implies the validity of the complete set of the bosonic blackfold equations (4.15), (4.16). More specifically, since the equations in the above parenthesis are the 'constraint' part of the independent subset of supergravity equations, it is natural to anticipate that by satisfying them we guarrantee also a solution of the full first order supergravity equations. Assuming this is correct, we deduce via (3.7) that the Einstein equations are also satisfied. That would imply that the corresponding extra constraint equations involving the effective stress-energy momentum conservation are also satisfied.

\section{Killing spinor equations: intrinsic deformations of M2-M5}

The intrinsic perturbations are monopole deformations with respect to the transverse foursphere. We do not perturb the transverse scalars $X^{\perp}$, and working locally around a point (call it $\sigma=0$ ) in Riemann normal coordinates we can also take the induced metric to be flat $\gamma_{a b}=\eta_{a b}$ [33]. The expansion is organized by the number of worldvolume derivatives, counted here by the power of the dummy variable $\boldsymbol{\delta}$. Working up to $\mathcal{O}(\boldsymbol{\delta})$ we set

$$
\begin{aligned}
r_{H}(\sigma) & =r_{H}(0)+\boldsymbol{\delta} \sigma^{a} \partial_{a} r_{H}+\mathcal{O}\left(\boldsymbol{\delta}^{2}\right), \quad \theta(\sigma)=\theta(0)+\boldsymbol{\delta} \sigma^{a} \partial_{a} \theta+\mathcal{O}\left(\boldsymbol{\delta}^{2}\right) \\
u^{0}(\sigma) & =1+\mathcal{O}\left(\boldsymbol{\delta}^{2}\right), \quad u^{b}(\sigma)=\boldsymbol{\delta} \sigma^{a} \partial_{a} u^{b}, \quad b=1,2,3,4,5 \\
v^{1}(\sigma) & =1+\mathcal{O}\left(\boldsymbol{\delta}^{2}\right), \quad v^{b}(\sigma)=\boldsymbol{\delta} \sigma^{a} \partial_{a} v^{b}, \quad b=0,2,3,4,5 \\
w^{2}(\sigma) & =1+\mathcal{O}\left(\boldsymbol{\delta}^{2}\right), \quad w^{b}(\sigma)=\boldsymbol{\delta} \sigma^{a} \partial_{a} w^{b}, \quad b=0,1,3,4,5
\end{aligned}
$$

Three additional relations are satisfied by the first derivative corrections of the worldvolume vectors $u, v, w$ as a result of the orthonormality conditions

$$
\begin{aligned}
u^{a} v_{a}=0 & \Rightarrow \partial_{a} v_{0}=\partial_{a} u_{1} \\
u^{a} w_{a}=0 & \Rightarrow \partial_{a} w_{0}=\partial_{a} u_{2}, \\
v^{a} w_{a}=0 & \Rightarrow \partial_{a} w_{1}=-\partial_{a} v_{2}
\end{aligned}
$$

The corrections $h_{\mu \nu}$ and $G_{4}$ are $\sigma$-independent monopoles on the transverse sphere. 
These data fix the form of the first order perturbation of the bosonic fields (4.10), (4.11), which we repeat here for convenience

$$
\begin{aligned}
d s^{2} & =\left(e^{-\frac{S}{3}} \hat{h}_{a b}+e^{-\frac{Q}{3}} \hat{\perp}_{a b}\right) d \sigma^{a} d \sigma^{b}+e^{-\frac{R}{3}}\left(r^{-2} d r^{2}+d \Omega_{4}^{2}\right)+\boldsymbol{\delta} h_{\mu \nu} d x^{\mu} d x^{\nu}+\mathcal{O}\left(\boldsymbol{\delta}^{2}\right), \\
F_{4} & =d \mathcal{C}_{3}+D^{-1} \star d \mathcal{C}_{6}+\boldsymbol{\delta} G_{4}+\mathcal{O}\left(\boldsymbol{\delta}^{2}\right), \\
\mathcal{C}_{3} & =-\sin \theta\left(H^{-1}-1\right) \omega_{3}-\tan \theta D H^{-1} *_{6} \omega_{3}, \quad \mathcal{C}_{6}=\cos \theta D\left(H^{-1}-1\right) \omega_{6} .
\end{aligned}
$$

Notice that these expressions contain implicit $\mathcal{O}(\boldsymbol{\delta})$ contributions that arise from the $\sigma$ expansion of the functions $S, Q, R, \hat{h}_{a b}, \mathcal{C}_{3}, \mathcal{C}_{6}$.

The supersymmetric subset of these deformations obeys the Killing spinor equations (3.5). For such configurations the Killing spinor $\eta$, which at zeroth order is a function of the radial coordinate $r$ parametrized by the constants $r_{H}, \theta$, is perturbed accordingly to

$$
\eta\left(r, \sigma, y^{i}\right)=\eta_{0}+\boldsymbol{\delta} \sigma^{a} \partial_{a} \eta\left(r, 0, y^{i}\right)+\boldsymbol{\delta} \zeta\left(r, y^{i}\right)+\mathcal{O}\left(\boldsymbol{\delta}^{2}\right)=\chi(r, \sigma) \otimes \xi\left(y^{i}\right)
$$

We used the notation $\eta_{0} \equiv \eta\left(r, 0, y^{i}\right)$ and $\zeta\left(r, y^{i}\right)$ is the fermionic analog of the corrections $h_{\mu \nu}, G_{4}$ that are needed to satisfy the full set of Killing spinor equations. $\xi$ is a Killing spinor on the unit $S^{4}(3.22)$ with $C= \pm 1$.

A straightforward computation reveals that the $\mathcal{O}(\boldsymbol{\delta})$ Killing spinor equations split into two groups. The first comes from the $\sigma$-independent part

$$
\begin{aligned}
\partial_{a} \eta+\bar{\Pi}_{1}^{(a)} \eta_{0} & =0, \\
\partial_{r} \zeta+\bar{\Pi}_{3} \eta_{0}+\Pi_{4} \zeta & =0, \\
\left(C \bar{\Pi}_{5}^{(m)}+\bar{\Pi}_{7}^{(m)}\right) \eta_{0}+\left(C \Pi_{6}^{(m)}+\Pi_{8}^{(m)}\right) \zeta & =0,
\end{aligned}
$$

where $m$ is an $S^{4}$ index. The form of the operators $\bar{\Pi}_{1}^{(a)}, \bar{\Pi}_{3}, \ldots$ is summarized in appendix A.1 .

The second group comes from the $\sigma$-linear piece of the Killing spinor equations

$$
\begin{aligned}
\Pi_{1, a}^{(b)} \eta_{0}+\Pi_{2}^{(b)} \partial_{a} \eta & =0, \\
\partial_{r} \partial_{a} \eta+\Pi_{3, a} \eta_{0}+\Pi_{4} \partial_{a} \eta & =0, \\
\left(C \Pi_{5, a}^{(m)}+\Pi_{7, a}^{(m)}\right) \eta_{0}+\left(C \Pi_{6}^{(m)}+\Pi_{8}^{(m)}\right) \partial_{a} \eta & =0 .
\end{aligned}
$$

The form of the operators $\Pi_{1, a}^{(b)}, \Pi_{2}^{(b)}, \ldots$ is summarized in appendix A.1. In contrast to the first group these equations do not involve the corrections $h_{\mu \nu}, G_{4}$, and therefore have the right features to play the Killing spinor counterpart of the bosonic constraint equations that give rise to the blackfold equations.

\section{$5.1 \kappa$-symmetry condition for intrinsic perturbations}

In section 3.3 we described how the analysis of the $a=0,1, \ldots, 5$ components of the supergravity Killing spinor equation for the zeroth-order solution produces at the asymptotic infinity a $\kappa$-symmetry-like projection equation (3.33) that can be mapped to the abelian 
PST $\kappa$-symmetry equation (2.12), (2.13). For the perturbed system the analogous components of the Killing spinor equation are (5.12), (5.15). We proceed to show how the $\sigma$-linear subset (5.15) produces a condition that will be mapped later to a perturbative version of the abelian PST $\kappa$-symmetry equation (2.6), (2.7). We have checked that the remaining $\sigma$-linear equations (5.16), (5.17) do not give rise to additional constraints on the Killing spinor at leading order in the $1 / r$ expansion that we are considering.

In what follows it will be convenient to establish the notation $a_{\|}$for worldvolume indices in the range $(0,1,2)$ and $a_{\perp}$ for indices in the range $(3,4,5)$.

Expanding the operators $\Pi_{2}^{(b)}, \Pi_{1, a}^{(b)}$ around the asymptotic infinity in the overlap region we find that the leading behavior is $\mathcal{O}\left(r^{-4}\right)$. Specifically,

$$
\begin{aligned}
& \Pi_{2}^{\left(b_{\|}\right)}=\frac{r_{H}^{3}(0)}{4 r^{4}} \Gamma_{\hat{b}_{||}} \Gamma_{\hat{r}}\left(1+\sin ^{2} \theta(0)+2 \sin \theta(0) \Gamma_{\|}\right. \\
& \left.+\sin \theta(0) \cos \theta(0) \Gamma_{\perp}+\cos \theta(0) \Gamma_{\hat{r}} \Gamma_{\Omega}\right)+\mathcal{O}\left(r^{-7}\right), \\
& \Pi_{2}^{\left(b_{\perp}\right)}=\frac{r_{H}^{3}(0)}{4 r^{4}} \Gamma_{\hat{b}_{\perp}} \Gamma_{\hat{r}}\left(1-2 \sin ^{2} \theta(0)-\sin \theta(0) \Gamma_{\|}\right. \\
& \left.-2 \sin \theta(0) \cos \theta(0) \Gamma_{\perp}+\cos \theta(0) \Gamma_{\hat{r}} \Gamma_{\Omega}\right)+\mathcal{O}\left(r^{-7}\right),
\end{aligned}
$$

and

$$
\begin{aligned}
\Pi_{1, a}^{\left(b_{\|}\right)} \eta_{0}= & \frac{1}{4 r^{4}} \delta_{b_{\| \mid}}^{\hat{b}_{\| \mid}} \Gamma_{\hat{b}_{\| \mid}} \Gamma_{\hat{r}} \times \\
& \left\{\partial_{a}\left(\left(1+\sin ^{2} \theta\right) r_{H}^{3}\right)+\partial_{a}\left(\cos \theta r_{H}^{3}\right) \Gamma_{\| \mid} \Gamma_{\perp}+2 \partial_{a}\left(\sin \theta r_{H}^{3}\right) \Gamma_{\|}\right. \\
& +\partial_{a}\left(\sin \theta \cos \theta r_{H}^{3}\right) \Gamma_{\perp}+(1+C \cos \theta(0))(-2+C \cos \theta(0)) r_{H}^{3}(0) \times \\
& \left.\times \sum_{c_{\perp}} \delta_{\hat{c}_{\perp}}^{c_{\perp}}\left(-\partial_{a} u_{c_{\perp}} \Gamma^{\hat{0} \hat{c}_{\perp}}+\partial_{a} v_{c_{\perp}} \Gamma^{\hat{1} \hat{c}_{\perp}}+\partial_{a} w_{c_{\perp}} \Gamma^{\hat{2} \hat{c} \hat{c}_{\perp}}\right)\right\} \eta_{0} \\
+ & \mathcal{O}\left(r^{-7}\right), \\
\Pi_{1, a}^{\left(b_{\perp}\right)} \eta_{0}= & \frac{1}{4 r^{4}} \delta_{b_{\perp}}^{\hat{b}_{\perp}} \Gamma_{\hat{b}_{\perp}} \Gamma_{\hat{r}} \times \\
& \left\{\partial_{a}\left(\left(1-2 \sin { }^{2} \theta\right) r_{H}^{3}\right)+\partial_{a}\left(\cos \theta r_{H}^{3}\right) \Gamma_{\|} \Gamma_{\perp}-\partial_{a}\left(\sin \theta r_{H}^{3}\right) \Gamma_{\|}\right. \\
& -2 \partial_{a}\left(\cos \theta \sin \theta r_{H}^{3}\right) \Gamma_{\perp}+(1+C \cos \theta(0))(1-2 C \cos \theta(0)) r_{H}^{3}(0) \times \\
& \left.\times \sum_{c_{\perp}} \delta_{\hat{c}_{\perp}}^{c_{\perp}}\left(-\partial_{a} u_{c_{\perp}} \Gamma^{\hat{0} \hat{c}_{\perp}}+\partial_{a} v_{c_{\perp}} \Gamma^{\hat{1} \hat{c} \hat{c}_{\perp}}+\partial_{a} w_{c_{\perp}} \Gamma^{\hat{2} \hat{c} \hat{c}_{\perp}}\right)\right\} \eta_{0} \\
+ & \mathcal{O}\left(r^{-7}\right) .
\end{aligned}
$$

Significant simplifications to the final expressions of $\Pi_{1, a}^{(b)} \eta_{0}$ were possible with the repeated use of the zeroth order equations (3.30), (3.33)

$$
\Gamma_{\|} \Gamma_{\perp} \eta_{0}=C \eta_{0}, \quad\left(1+C \cos \theta(0)+\sin \theta(0) \Gamma_{\|}\right) \eta_{0}=0
$$


We notice that the explicit dependence on the index $b_{\|}$and $b_{\perp}$ has disappeared in the quantities $\Gamma^{\hat{b}_{\|}} \Pi_{1, a}^{\left(b_{\|}\right)} \eta_{0}, \Gamma^{\hat{b}_{\perp}} \Pi_{1, a}^{\left(b_{\perp}\right)} \eta_{0}$. Then, by taking, for example, the linear combination ${ }^{7}$

$$
2 \Gamma_{\hat{r}} \Gamma^{\hat{b}_{\|}} \times\left((5.15) \text { with } b=b_{\|}\right)+\Gamma_{\hat{r}} \Gamma^{\hat{b}_{\perp}} \times\left((5.15) \text { with } b=b_{\perp}\right)
$$

(and isolating the leading $\mathcal{O}\left(r^{-4}\right)$ terms) we discover the single independent condition from these equations

$$
\Pi_{1, a} \eta_{0}+\Pi_{2} \partial_{a} \eta=0
$$

where

$$
\begin{aligned}
\Pi_{1, a}= & \partial_{a} r_{H}^{3}+\partial_{a}\left(\cos \theta r_{H}^{3}\right) \Gamma_{\|} \Gamma_{\perp}+\partial_{a}\left(\sin \theta r_{H}^{3}\right) \Gamma_{\|} \\
& -(1+C \cos \theta(0)) r_{H}^{3}(0) \sum_{c_{\perp}} \delta_{\hat{c}_{\perp}}^{c_{\perp}}\left(-\partial_{a} u_{c_{\perp}} \Gamma^{\hat{0} \hat{c}_{\perp}}+\partial_{a} v_{c_{\perp}} \Gamma^{\hat{1} \hat{c}_{\perp}}+\partial_{a} w_{c_{\perp}} \Gamma^{\hat{2} \hat{c}_{\perp}}\right) \\
\Pi_{2}= & r_{H}^{3}(0)\left(1+\sin \theta(0) \Gamma_{\|}+\cos \theta(0) \Gamma_{\|} \Gamma_{\perp}\right) .
\end{aligned}
$$

Recall that $\Pi_{2} \eta_{0}=0$ is the zeroth order Killing spinor equation (3.33). A simple consistency check of (5.23)-(5.25) is performed in appendix B.1. We have also checked that these equations reproduce the expected 1/4-BPS supersymmetry of the self-dual string soliton solution of ref. [23].

The last central observation is that (5.23) can be recast as a perturbative version of the abelian PST $\kappa$-symmetry equation (2.6), (2.7) with a specific mapping between gauge and gravity variables. For starters, let us set

$$
\epsilon(\sigma)=f(\sigma) \eta(\sigma)
$$

for the relation between the spinors appearing in equations (2.6) and (5.23). The relative, generally $\sigma$-dependent, factor $f(\sigma)$ will be fixed in a moment. Expanding around $\sigma=0$ we then have locally

$$
\epsilon(\sigma)=f(0) \eta_{0}+\boldsymbol{\delta} \sigma^{a}\left(f(0) \partial_{a} \eta+\partial_{a} f \eta_{0}\right)+\mathcal{O}\left(\boldsymbol{\delta}^{2}\right)
$$

Now consider an intrinsic fluctuation of the abelian PST $\kappa$-symmetry matrix around the zeroth order profile (2.9), (2.10). In this deformation the originally constant fields $\mathbf{v}_{a}, \tilde{H}_{a b}$ become slowly-varying along the worldvolume, but the transverse scalars are kept constant and the worldvolume metric flat. Then, $\boldsymbol{\Gamma}_{\kappa}$ perturbs to

$$
\boldsymbol{\Gamma}_{\kappa}(\sigma)=\boldsymbol{\Gamma}_{\kappa}(0)+\boldsymbol{\delta} \sigma^{a} \boldsymbol{\Gamma}_{\kappa, a}+\mathcal{O}\left(\boldsymbol{\delta}^{2}\right)
$$

where $\boldsymbol{\Gamma}_{\kappa}(0)$ is given by $(2.11)$ and

$$
\begin{aligned}
\boldsymbol{\Gamma}_{\kappa, a}= & \partial_{a} \boldsymbol{\Gamma}_{\kappa}=\partial_{a}\left(\frac{\mathbf{v}_{b} t_{c}}{\sqrt{-\operatorname{det}(\eta+\tilde{H})}}\right) \Gamma^{b c}+ \\
& +\partial_{a}\left(\frac{\mathbf{v}_{b} \tilde{H}_{c d}}{2 \sqrt{-\operatorname{det}(\eta+\tilde{H})}}\right) \Gamma^{b c d}-\partial_{a}\left(\frac{1}{\sqrt{-\operatorname{det}(\eta+\tilde{H})}}\right) \Gamma_{||} \Gamma_{\perp} .
\end{aligned}
$$

\footnotetext{
${ }^{7}$ We remind that the same combinations were considered at zeroth order.
} 
Notice that there is no $\Gamma_{\perp}$ contribution in this expression. In addition, two possible contributions to the last term from derivatives of the vector $\mathbf{v}$ cancel each other out.

Combining the expansions (5.27), (5.28), we find that the $\kappa$-symmetry equation (2.6) is, by definition, satisfied at zeroth order. At first order we obtain

$$
\left(f(0) \boldsymbol{\Gamma}_{\kappa, a}+\left(\boldsymbol{\Gamma}_{\kappa}(0)-1\right) \partial_{a} f\right) \eta_{0}+f(0)\left(\boldsymbol{\Gamma}_{\kappa}(0)-1\right) \partial_{a} \eta=0 .
$$

We conclude that a map between the perturbative field theory Killing spinor equation (5.30) and the supergravity induced one (5.23) is possible if we can set ${ }^{8}$

$$
\Pi_{1, a} \eta_{0}=\left(f(0) \boldsymbol{\Gamma}_{\kappa, a}+\left(\boldsymbol{\Gamma}_{\kappa}(0)-1\right) \partial_{a} f\right) \eta_{0}, \quad \Pi_{2}=f(0)\left(\boldsymbol{\Gamma}_{\kappa}(0)-1\right) .
$$

One of the first checks is the absence of $\Gamma_{\perp}$ terms both in (5.23) and (5.30). Other terms, e.g. terms proportional to the identity, also work properly. All potentially harmful terms that can spoil the match (5.31) cancel out at the end of the computation. Equations (5.31) can be satisfied by requiring the gauge-gravity map

$$
\begin{aligned}
\partial_{a} f & =-\partial_{a}\left(r_{H}^{3}\right), \\
\partial_{a}\left(\frac{1}{\sqrt{-\operatorname{det}(\eta+\tilde{H})}}\right) & =\partial_{a}(\cos \theta), \\
\partial_{a}\left(-\frac{1}{3 !} \varepsilon^{a_{\|} b_{\|} c_{\|} \mathbf{v}_{a_{\|}} \tilde{H}_{b_{\|} c_{||}}}\right) & =\partial_{a}(\tan \theta(\sigma)), \\
\sin \theta(0) \partial_{a} \mathbf{v}_{c_{\perp}}+\frac{1}{2} \varepsilon_{c_{\perp}}^{b_{\perp} d_{\perp}} \partial_{a} \tilde{H}_{b_{\perp} d_{\perp}} & =-\sin \theta(0) \partial_{a} u_{c_{\perp}}, \\
\partial_{a} \tilde{H}_{2 c_{\perp}} & =\tan \theta(0) \partial_{a} v_{c_{\perp}}, \\
\partial_{a} \tilde{H}_{1 c_{\perp}} & =-\tan \theta(0) \partial_{a} w_{c_{\perp}} .
\end{aligned}
$$

We observe that there are some field components on both sides that do not appear in this map, i.e. are not needed in order to match the $\kappa$-symmetry conditions. On the abelian PST side these fields are two of the $b_{\perp}$ components of $\partial_{a} \tilde{H}_{0 b_{\perp}}$. On the supergravity/blackfold side the derivatives $\partial_{a} u_{b_{||}}, \partial_{a} v_{b_{\|}}, \partial_{a} w_{b_{\|}}$(of which only two are independent, see (5.5)-(5.7)) do not appear. As we noted at the end of subsection 4.3, however, it is anticipated that the full set of bosonic blackfold equations follows from the combination of the Killing spinor equations and the charge current conservation equations. In general, the 'missing' components will appear in these equations explicitly. Analogous statements apply to the abelian PST side [3].

\section{Killing spinor equations: extrinsic deformations of M2-M5}

A similar analysis can be performed for extrinsic deformations of the planar M2-M5 solution. In this case the intrinsic variables $-r_{H}, \theta$ and the vectors $u, v, w-$ remain unperturbed. The deformation activates the transverse scalars $X^{\perp}$ and perturbs accordingly the

\footnotetext{
${ }^{8}$ Since $\eta_{0}$ is a special spinor obeying the zeroth order Killing spinor equation, it is important in the first equality to keep the action on it explicit. Then, by using the zeroth order equations we can manipulate the form of the first order equations appropriately.
} 
induced worldvolume metric. As explained in detail in [33], it is convenient to work in a local adapted coordinate system employing Fermi normal coordinates. This system assigns coordinates $\left(\sigma^{a}, z^{i}\right),(i=6, \ldots, 10)$, to the point that lies a unit affine distance along the geodesic with tangent $\frac{\partial}{\partial z^{i}}$ orthogonally to the worldvolume at $\sigma^{a}$. Then, perturbations around a locally flat worldvolume patch are induced by the extrinsic curvature tensor $K_{a b}{ }^{i}$ along each of the transverse directions $z^{i}$. The linear independence of these perturbations for each $i$ implies that we can set all but one to zero and study them independently. Accordingly, we introduce a director cosine

$$
z^{i}=r \cos \phi
$$

for a fixed $i$ and denote for convenience $K_{a b}{ }^{i} \equiv K_{a b}$. Following [33] we can then bring the first order dipole deformation of the metric (in the near-zone region) into the form

$$
\begin{aligned}
d s^{2}= & \left(e^{-\frac{S}{3}} \hat{h}_{a b}+e^{-\frac{Q}{3}} \hat{\perp}_{a b}-2 \boldsymbol{\delta} K_{a b} r \cos \phi\right) d \sigma^{a} d \sigma^{b} \\
& +e^{-\frac{R}{3}}\left(r^{-2} d r^{2}+d \phi^{2}+\sin ^{2} \phi d \Omega_{3}^{2}\right)+\boldsymbol{\delta} h_{\mu \nu}(r, \phi) d x^{\mu} d x^{\nu}+\mathcal{O}\left(\boldsymbol{\delta}^{2}\right) .
\end{aligned}
$$

The projector $\hat{h}_{a b}$ is not perturbed. For convenience, in what follows we set $\hat{h}=$ $\operatorname{diag}(-1,1,1,0,0,0)$. The orthogonal projector is $\hat{\perp}_{a b}=\eta_{a b}-\hat{h}_{a b}$. The functions $S, Q, R$ are not perturbed and are given by the zeroth order expressions (3.17).

For the perturbed 4 -form field strength we propose the ansatz

$$
F_{4}=d \mathcal{C}_{3}+D^{-1} \star d \mathcal{C}_{6}+\boldsymbol{\delta} G_{4}(r, \phi)+\mathcal{O}\left(\boldsymbol{\delta}^{2}\right)
$$

where again

$$
\mathcal{C}_{3}=-\sin \theta\left(H^{-1}-1\right) \omega_{3}-\tan \theta D H^{-1} *_{6} \omega_{3}, \quad \mathcal{C}_{6}=\cos \theta D\left(H^{-1}-1\right) \omega_{6} .
$$

The form $\omega_{3}$ (4.14) is not perturbed, but $\omega_{6}$ (4.14) is perturbed in accordance with the worldvolume metric deformation

$$
\gamma_{a b}=\eta_{a b}-2 \boldsymbol{\delta} K_{a b} r \cos \phi .
$$

Similarly, $*_{6}$ is perturbed according to (6.5).

Compared to the previous section of intrinsic perturbations, now the bosonic corrections $h_{\mu \nu}$ and $G_{4}$ are dipole perturbations in the transverse sphere.

For supersymmetric configurations the Killing spinor $\eta$ receives a corresponding dipole perturbation

$$
\begin{aligned}
\eta\left(r, \sigma, \phi, \vartheta^{m}\right) & =\eta_{0}+\boldsymbol{\delta} \eta_{1} \\
& =\eta_{0}+\boldsymbol{\delta} \cos \phi\left(\lambda\left(r, \vartheta^{m}\right)+r K_{a b}(\sigma) \xi^{a b}\left(r, \vartheta^{m}\right)\right)+\mathcal{O}\left(\boldsymbol{\delta}^{2}\right) \\
& =\eta_{0}+\chi(r, \phi) \otimes \psi\left(\vartheta^{m}\right) .
\end{aligned}
$$

$\vartheta^{m}(m=1,2,3)$ are coordinates on the unit $S^{3} . \eta_{0}$ is the zeroth order Killing spinor and $\eta_{1}$ its first order correction. In analogy to the previous discussion, we have separated the 
contributions to $\eta_{1}$ into a piece $\xi^{a b}$ induced directly by the extrinsic curvature, and a second piece $\lambda$ needed to satisfy the full set of Killing spinor equations. $\lambda$ is also first order and proportional to $K_{a b}$ but does not vanish as $r \rightarrow 0$. Since we break the transverse $\mathrm{SO}(5)$ symmetry, but retain an $\mathrm{SO}(4)$ subset, we are now expressing the spinor correction $\eta_{1}$ as a tensor product with a unit $S^{3}$ Killing spinor $\psi$, whose covariant derivative on $S^{3}$ is by definition

$$
\nabla_{m} \psi=\frac{i \tilde{C}}{2} \Gamma_{m} \psi, \quad \tilde{C}= \pm 1 .
$$

In this particular equation $\Gamma_{m}=e_{m}^{\hat{m}} \Gamma_{\hat{m}}$ where $e_{m}^{\hat{m}}$ is the vielbein in the unit $S^{3}$. We note that $\eta_{0}$ is instead an $S^{4}$ Killing spinor with an a priori independent $\operatorname{sign} C$ (3.22).

Inserting this ansatz into the Killing spinor equations (3.5) we find the following set of perturbative spinor equations

$$
\begin{aligned}
\Sigma_{1}^{(a)} \eta_{0}+\Sigma_{2}^{(a)} \eta_{1} & =0, \\
\partial_{r} \eta_{1}+\Sigma_{3} \eta_{0}+\Sigma_{4} \eta_{1} & =0, \\
\partial_{\phi} \eta_{1}+\Sigma_{5} \eta_{0}+\Sigma_{6} \eta_{1} & =0, \\
\left(C \Sigma_{7}^{(m)}+\Sigma_{9}^{(m)}\right) \eta_{0}+\left(\tilde{C} \Sigma_{8}^{(m)}+\Sigma_{10}^{(m)}\right) \eta_{1} & =0 .
\end{aligned}
$$

The more explicit form of the operators $\Sigma_{i}$ is summarized in appendix A.2. When we implement on $\eta_{1}$ the ansatz of the second line in (6.6) we find as in section 5 that the equations split into two groups, which are required to hold independently. The first group does not receive any contributions from terms linear in $r K_{a b}$, but involves explicitly the corrections $h_{\mu \nu}, G_{4}$ and $\lambda$. The second group depends linearly on $r K_{a b}$, but not on the corrections $h_{\mu \nu}, G_{4}, \lambda$. We proceed to analyze some of the implications of the second group.

\section{1 $\kappa$-symmetry condition for extrinsic perturbations}

In direct analogy with the approach followed in section 5 , we concentrate on the $\left(r K_{a b}\right)$ dependent part of the first set of perturbative Killing spinor equations (6.8). Expanding the operators $\Sigma_{1}^{(a)}, \Sigma_{2}^{(a)}$ around the asymptotic infinity in the overlap region we find the leading order equations

$$
K_{a b}\left(\Sigma_{1}^{a b(c)} \eta_{0}+\Sigma_{2}^{(c)} \xi^{a b}\right)=0
$$

where

$$
\begin{gathered}
K_{b c} \Sigma_{1}^{b c\left(a_{\|}\right)} \eta_{0}=\cos \phi \frac{r_{H}^{3}}{r^{3}} \delta_{a_{\| \mid}}^{\hat{a}_{\|}} \Gamma_{\hat{a}_{\|}} \Gamma_{\hat{r}}\left[-\frac{3}{2} \sin \theta \cos \theta \hat{\Gamma}+\frac{1}{4} K\left(\sin \theta \cos \theta \Gamma_{\perp}-\cos \theta \Gamma_{\|} \Gamma_{\perp}\right)\right. \\
\left.-\frac{1}{4} \sin \theta \eta^{d d} K_{d a}\left(\omega_{3}\right)_{d b c} \Gamma^{a b c}-\frac{1}{8} \sin \theta \cos \theta \eta^{d d} K_{d a}\left(*_{\eta} \omega_{3}\right)_{d b c} \Gamma^{a b c}\right] \eta_{0}+\mathcal{O}\left(r^{-6}\right), \\
K_{b c} \Sigma_{1}^{b c\left(a_{\perp}\right)} \eta_{0}=\cos \phi \frac{r_{H}^{3}}{r^{3}} \delta_{a_{\perp}}^{\hat{a}_{\perp}} \Gamma_{\hat{a}_{\perp}} \Gamma_{\hat{r}}\left[3 \sin \theta \cos \theta \hat{\Gamma}+\frac{1}{4} K\left(-2 \sin \theta \cos \theta \Gamma_{\perp}-\cos \theta \Gamma_{\|} \Gamma_{\perp}\right)\right. \\
\left.+\frac{1}{8} \sin \theta \eta^{d d} K_{d a}\left(\omega_{3}\right)_{d b c} \Gamma^{a b c}+\frac{1}{4} \sin \theta \cos \theta \eta^{d d} K_{d a}\left(*_{\eta} \omega_{3}\right)_{d b c} \Gamma^{a b c}\right] \eta_{0}+\mathcal{O}\left(r^{-6}\right),
\end{gathered}
$$

and

$$
\Sigma_{2}^{(c)}=r \cos \phi \Pi_{2}^{(c)}
$$


$\Pi_{2}^{(c)}$ is the operator that appears already in equations (5.18), (5.19). In (6.13), (6.14) we used the notation

$$
K \equiv \eta^{a b} K_{a b}, \quad \hat{\Gamma} \equiv \frac{1}{3 !} \varepsilon_{a b c d_{1} d_{2} d_{3}}\left(\omega_{3}\right)_{e_{1} e_{2} e_{3}} \eta^{d_{1} e_{1}} \eta^{d_{2} e_{2}} K^{d_{3} e_{3}} \Gamma^{a b c}
$$

Superficially, the leading order contributions to $K_{b c} \Sigma_{1}^{b c(a)} \eta_{0}$ are order $\mathcal{O}(r)$. The total cancellation of these dangerous contributions is due to the identity (see appendix B.2 for an explicit derivation)

$$
\left(\cos \phi \Gamma_{\hat{r}}-\sin \phi \Gamma_{\hat{\phi}}\right) \eta_{0}=0
$$

which follows essentially from the fact that $\eta_{0}$ is an $S^{4}$ Killing spinor. Further important cancellations occur at the next order $\mathcal{O}\left(r^{-3}\right)$ because of $(6.17)$ and the zeroth order equation (3.33).

As in section 5.1 we consider the linear combination

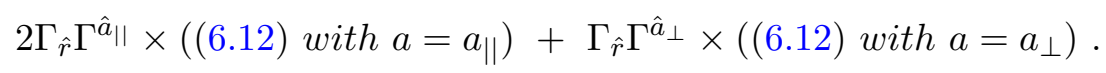

Isolating the leading $\mathcal{O}\left(r^{-3}\right)$ terms we arrive at a single $\left(a_{\|}, a_{\perp}\right)$-independent equation of the form

$$
K_{a b}\left(\Sigma_{1}^{a b} \eta_{0}+\Pi_{2} \xi^{a b}\right)=0
$$

with

$$
\begin{aligned}
K_{a b} \Sigma_{1}^{a b} & =-r_{H}^{3}\left(K \cos \theta \Gamma_{\|} \Gamma_{\perp}+\frac{1}{2} \sin \theta \eta^{d d} K_{d a}\left(\omega_{3}\right)_{d b c} \Gamma^{a b c}\right), \\
\Pi_{2} & =r_{H}^{3}\left(1+\sin \theta \Gamma_{\|}+\cos \theta \Gamma_{\|} \Gamma_{\perp}\right) .
\end{aligned}
$$

We can now show that this equation is the same as the $\kappa$-symmetry equation (2.6) of the abelian PST theory perturbed around the constant- $\mathcal{H}$ flux solution (2.9), (2.10). For extrinsic deformations (restricted to a specific transverse space direction $i$ for which $\left.X_{i}=X\right)$ the PST Killing spinor $\epsilon$ perturbs to

$$
\epsilon=\epsilon_{0}+\boldsymbol{\delta} X K_{a b} \epsilon^{a b}+\mathcal{O}\left(\boldsymbol{\delta}^{2}\right)=-r_{H}^{3}\left(\eta_{0}-\boldsymbol{\delta} X K_{a b} \xi^{a b}+\mathcal{O}\left(\boldsymbol{\delta}^{2}\right)\right)
$$

where in the second equality we used the map

$$
\epsilon_{0}=-r_{H}^{3} \eta_{0}, \quad \epsilon^{a b}=r_{H}^{3} \xi^{a b}
$$

At the same time, the $\kappa$-symmetry matrix $\boldsymbol{\Gamma}_{\kappa}(2.7)$ perturbs to

$$
\boldsymbol{\Gamma}_{\kappa}(X)=\boldsymbol{\Gamma}_{\kappa}^{(0)}+\boldsymbol{\delta} \delta \boldsymbol{\Gamma}_{\kappa}+\ldots
$$

The zeroth order term is $\Gamma_{\kappa}^{(0)}=-\left(\sin \theta \Gamma_{\|}+\cos \theta \Gamma_{||} \Gamma_{\perp}\right)$ (see (2.11), (2.12)). The first order term $\delta \boldsymbol{\Gamma}_{\kappa}$ is induced by the metric perturbation

$$
\delta \gamma_{a b}=\gamma_{a b}-\eta_{a b}=-2 \boldsymbol{\delta} X K_{a b},
$$


at fixed 'intrinsic' fields $\mathbf{v}_{a}, \tilde{H}_{a b}$. Using the profile of the zeroth order solution along with the definitions (2.12), and the variation identities

$$
\begin{aligned}
\delta\left(\frac{1}{\sqrt{-\operatorname{det}(\gamma+\tilde{H})}}\right) & =-\frac{1}{2} \frac{1}{\sqrt{-\operatorname{det}(\gamma+\tilde{H})}} \gamma^{a b} \delta \gamma_{a b}=\cos \theta X K, \\
\delta\left(\frac{\sqrt{-\operatorname{det} \gamma}}{2 \sqrt{-\operatorname{det}(\gamma+\tilde{H})}} \mathbf{v}_{a} \tilde{H}_{b c} e_{\hat{a}}^{a} e_{\hat{b}}^{b} e_{\hat{c}}^{c} \Gamma^{\hat{a} \hat{b} \hat{c}}\right) & =-\frac{1}{2} \sin \theta \eta^{d d} X K_{d a}\left(\omega_{3}\right)_{d b c} \Gamma^{a b c}
\end{aligned}
$$

we obtain

$$
\delta \boldsymbol{\Gamma}_{\kappa}=-\frac{1}{2} \sin \theta \eta^{d d} K_{d a}\left(\omega_{3}\right)_{d b c} \Gamma^{a b c}-\cos \theta K \Gamma_{\|} \Gamma_{\perp} .
$$

Assembling all the elements, the $\kappa$-symmetry equation (2.6) becomes

$$
\begin{aligned}
r_{H}^{3}[-(K \cos \theta & \Gamma_{\|} \Gamma_{\perp}+\frac{1}{2} \sin \theta \eta^{d d} K_{d a}\left(\omega_{3}\right) d b c \\
& \left.\Gamma^{a b c}\right) \eta_{0} \\
& \left.+\left(1+\sin \theta \Gamma_{\|}+\cos \theta \Gamma_{\|} \Gamma_{\perp}\right) K_{a b} \xi^{a b}\right]=0
\end{aligned}
$$

reproducing the supergravity equations (6.18)-(6.20).

\section{Open issues and outlook}

In this paper, following the lore of the blackfold approach [9], we have addressed the problem of long-wavelength supersymmetric deformations of M5 brane solutions in elevendimensional supegravity. Initiating a study of the leading order perturbation of the supergravity Killing spinor equations, we have shown that part of these equations gives rise to a perturbative $\kappa$-symmetry-like condition for the blackfold effective worldvolume theory. This equation exhibits the same structure as the $\kappa$-symmetry equation of the abelian PST theory of a single M5 brane. Requiring a match between the two we have obtained a nonlinear map between the fields of the PST theory and the supergravity-derived fields of the blackfold effective theory.

It would be very interesting to obtain a more covariant form of this map extending the local analysis of this paper and including the charge current conservation equations. This map would have several consequences. First, it would suggest an intriguing rewriting of the PST theory in a fluid-dynamical language. Could this lead to a fruitful reformulation of the theory on M5 branes? Second, it would provide deeper insight into the blackfold equations. New supersymmetric solutions can be envisioned by converting the second order bosonic blackfold equations to first order ones. Third, on a more conceptual level, this map would help elucidate a potential gauge-gravity equivalence for the full brane system in flat space.

The other important open problem is the full solution of the first order perturbed supergravity equations. For that purpose one has to consider the complete set of Killing spinor equations in the near-zone, extend the analysis to the far-zone $\left(r \gg r_{H}\right)$ and finally 
perform the match in the overlap zone $\left(r_{H} \ll r \ll \mathcal{R}\right)$. This would establish a concrete relation between supersymmetric solutions of the leading order blackfold equations and full first-order corrected regular supergravity solutions. We anticipate this is a one-to-one relation [9]. Progress in this problem may entail an educated use of the underlying $G$ structure [44] of the seed solution and its deformation. This prospect is currently under investigation. The higher orders of the expansion scheme are also of interest. The more constrained structures of supersymmetric solutions may lead in this context to a more tractable setup compared to the general non-supersymmetric, finite temperature situation.

Finally, although here we focused on M-theory and eleven-dimensional supergravity, it is natural to expect that analogous statements carry over to the brane solutions of other higher-dimensional supergravities. Branes in the ten-dimensional type IIA/B (and connections to the DBI theory) are an obvious context for future study.

\section{Acknowledgments}

I would like to thank C. Bachas, J. Camps, S. Katmadas, E. Kiritsis, N. Obers, G. Papadopoulos, A. Pedersen, K. Siampos, A. Tomasiello and A. Zaffaroni for enlightening discussions. This work was supported in part by European Union's Seventh Framework Programme under grant agreements (FP7-REGPOT-2012-2013-1) no 316165, PIF-GA2011-300984, the EU program "Thales" MIS 375734 and was also co-financed by the European Union (European Social Fund, ESF) and Greek national funds through the Operational Program "Education and Lifelong Learning" of the National Strategic Reference Framework (NSRF) under "Funding of proposals that have received a positive evaluation in the 3rd and 4th Call of ERC Grant Schemes".

\section{A Summary of perturbative Killing spinor equations}

In this appendix we summarize the full set of intrinsic and extrinsic perturbations of the Killing spinor equations (3.5). We present the raw structure of the equations omitting details that are not used in the main text.

\section{A.1 Intrinsic perturbations}

The intrinsic deformation of the metric $g_{\mu \nu}$ and four-form flux $F_{4}$ appears in eqs. (5.8)(5.10). For the metric correction $h_{\mu \nu}$ it is convenient to choose the gauge

$$
h_{r r}=0, h_{\mu y^{i}}=0
$$

where $y^{i}(i=1,2,3,4)$ are coordinates of the transverse $S^{4}$. Then, (5.8) takes the more specific form

$$
\begin{aligned}
d s^{2}= & \left(e^{-\frac{S}{3}} \hat{h}_{a b}+e^{-\frac{Q}{3}} \hat{\perp}_{a b}+\boldsymbol{\delta} h_{a b}\right) d \sigma^{a} d \sigma^{b}+2 \boldsymbol{\delta} h_{r a} d r d \sigma^{a} \\
& +r^{-2} e^{-\frac{R}{3}} d r^{2}+e^{-\frac{R}{3}}\left(1+\boldsymbol{\delta} h_{\Omega}\right) d \Omega_{4}^{2}+\mathcal{O}\left(\boldsymbol{\delta}^{2}\right) \\
= & d s_{0}^{2}+\boldsymbol{\delta} d s_{1}^{2}+\mathcal{O}\left(\boldsymbol{\delta}^{2}\right) .
\end{aligned}
$$


Do not confuse the projector $\hat{h}_{a b}$ with the metric perturbation $h_{a b}$. The components $h_{a b}$, $h_{r a}, h_{\Omega}$ are functions of the radial coordinate $r$ only. When the functions $S, Q, R, \hat{h}_{a b}$ are expanded in $\sigma$-derivatives the total $\mathcal{O}(\boldsymbol{\delta})$ contribution is collected in the first order correction $d s_{1}^{2}$. Accordingly, the vielbein components $e_{\nu}^{\hat{\mu}}$, and the components of the spin connection $\omega_{\mu}^{\hat{\nu} \hat{\rho}}$ are shifted to

$$
\begin{aligned}
e_{\nu}^{\hat{\mu}} & =\left(e_{0}\right)_{\nu}^{\hat{\mu}}+\boldsymbol{\delta}\left(e_{1}\right)_{\nu}^{\hat{\mu}}+\mathcal{O}\left(\boldsymbol{\delta}^{2}\right), \\
\omega_{\mu}^{\hat{\nu} \hat{\rho}} & =\eta^{\hat{\rho} \hat{\mu}} e_{\hat{\mu}}^{\nu} \partial_{[\nu} e_{\mu]}^{\hat{\nu}}-\eta^{\hat{\nu} \hat{\mu}} e_{\hat{\mu}}^{\nu} \partial_{[\nu} e_{\mu]}^{\hat{\rho}}+\eta^{\hat{\rho} \hat{\mu}} \eta^{\hat{\nu} \hat{\sigma}} \eta_{\hat{\tau} \hat{\lambda}} e_{\hat{\mu}}^{\nu} e_{\hat{\sigma}}^{\sigma} e_{\mu}^{\hat{\tau}} \partial_{[\nu} e_{\sigma]}^{\hat{\lambda}} \\
& =\left(\omega_{\mu}^{\hat{\nu} \hat{\rho}}\right)_{0}+\boldsymbol{\delta}\left(\omega_{\mu}^{\hat{\nu} \hat{\rho}}\right)_{1}+\mathcal{O}\left(\boldsymbol{\delta}^{2}\right) .
\end{aligned}
$$

We remind that the covariant derivatives of spinors are

$$
\nabla_{\mu}=\partial_{\mu}+\frac{1}{4} \omega_{\mu}^{\hat{\nu} \hat{\rho}} \Gamma_{\hat{\nu} \hat{\rho}}=\partial_{\mu}+\frac{1}{4}\left(\omega_{\mu}^{\hat{\nu} \hat{\rho}}\right)_{0} \Gamma_{\hat{\nu} \hat{\rho}}+\frac{1}{4}\left(\omega_{\mu}^{\hat{\nu} \hat{\rho}}\right)_{1} \Gamma_{\hat{\nu} \hat{\rho}} \boldsymbol{\delta}+\mathcal{O}\left(\boldsymbol{\delta}^{2}\right)
$$

After the implementation of the $\sigma$-expansion on the forms $\mathcal{C}_{3}$ and $\mathcal{C}_{6}$ in (5.9), (5.10), the four-form flux $F_{4}$ expands similarly to

$$
F_{4}=\left(F_{4}\right)_{0}+\boldsymbol{\delta}\left(F_{4}\right)_{1}+\mathcal{O}\left(\boldsymbol{\delta}^{2}\right) .
$$

For the slash

$$
F=\Gamma^{\nu_{1} \nu_{2} \nu_{3} \nu_{4}} F_{\nu_{1} \nu_{2} \nu_{3} \nu_{4}}
$$

we obtain

$$
\not \prime=F_{0}+\boldsymbol{\delta} \not f_{1}+\mathcal{O}\left(\boldsymbol{\delta}^{2}\right)
$$

where

$$
\begin{aligned}
& F_{0}=\left(\mathcal{P}_{0}\right)_{\hat{\mu}_{1} \nu_{2} \hat{\mu}_{2} \hat{\mu}_{3} \hat{\mu}_{4}}^{\nu_{2} \nu_{4}} \Gamma^{\hat{\mu}_{1} \hat{\mu}_{2} \hat{\mu}_{3} \hat{\mu}_{4}}\left(F_{\nu_{1} \nu_{2} \nu_{3} \nu_{4}}\right)_{0} \\
& F_{1}=\left(\mathcal{P}_{0}\right)_{\hat{\mu}_{1} \hat{\mu}_{2} \hat{\mu}_{3} \hat{\mu}_{4}}^{\nu_{2} \nu_{2} \nu_{3} \nu_{4}} \Gamma^{\hat{\mu}_{1} \hat{\mu}_{2} \hat{\mu}_{3} \hat{\mu}_{4}}\left(F_{\nu_{1} \nu_{2} \nu_{3} \nu_{4}}\right)_{1}+4\left(\mathcal{P}_{1}\right)_{\hat{\mu}_{1} \hat{\mu}_{2} \hat{\mu}_{3} \hat{\mu}_{4}}^{\nu_{2} \nu_{2} \nu_{3} \nu_{4}} \Gamma^{\hat{\mu}_{1} \hat{\mu}_{2} \hat{\mu}_{3} \hat{\mu}_{4}}\left(F_{\nu_{1} \nu_{2} \nu_{3} \nu_{4}}\right)_{0} .
\end{aligned}
$$

We used the shorthand notation

$$
\begin{aligned}
& \left(\mathcal{P}_{0}\right)_{\hat{\mu}_{1} \hat{\mu}_{2} \hat{\mu}_{3} \hat{\mu}_{4}}^{\nu_{1} \nu_{2} \nu_{3} \nu_{4}} \equiv\left(e_{0}\right)_{\hat{\mu}_{1}}^{\nu_{1}}\left(e_{0}\right)_{\hat{\mu}_{2}}^{\nu_{2}}\left(e_{0}\right)_{\hat{\mu}_{3}}^{\nu_{3}}\left(e_{0}\right)_{\hat{\mu}_{4}}^{\nu_{4}}, \\
& \left(\mathcal{P}_{1}\right)_{\hat{\mu}_{1} \hat{\mu}_{2} \hat{\mu}_{3} \hat{\mu}_{4}}^{\nu_{1} \nu_{2} \nu_{4}} \equiv\left(e_{0}\right)_{\hat{\mu}_{1}}^{\nu_{1}}\left(e_{0}\right)_{\hat{\mu}_{2}}^{\nu_{2}}\left(e_{0}\right)_{\hat{\mu}_{3}}^{\nu_{3}}\left(e_{1}\right)_{\hat{\mu}_{4}}^{\nu_{4}} .
\end{aligned}
$$

These expressions, together with the expansion of the Killing spinor (5.11)

$$
\eta=\eta_{0}+\boldsymbol{\delta} \eta_{1}+\mathcal{O}\left(\boldsymbol{\delta}^{2}\right)
$$

are then inserted into the Killing spinor equations (3.5)

$$
\nabla_{\mu} \eta+\frac{1}{288}\left(-\frac{1}{2} \Gamma_{\mu} \not H+\frac{3}{2} \not H \Gamma_{\mu}\right) \eta=0
$$

to obtain a set of $\mathcal{O}(\boldsymbol{\delta})$ equations of the form

$$
\begin{aligned}
\Pi_{1}^{(a)} \eta_{0}+\Pi_{2}^{(a)} \eta_{1} & =0 \\
\partial_{r} \eta_{1}+\Pi_{3} \eta_{0}+\Pi_{4} \eta_{1} & =0 \\
\left(C \Pi_{5}^{(m)}+\Pi_{7}^{(m)}\right) \eta_{0}+\left(C \Pi_{6}^{(m)}+\Pi_{8}^{(m)}\right) \eta_{1} & =0
\end{aligned}
$$


where $m$ is an $S^{4}$ index and

$$
\begin{aligned}
& \Pi_{1}^{(a)}=\frac{1}{4}\left(\omega_{a}^{\hat{\nu} \hat{\rho}}\right)_{1} \Gamma_{\hat{\nu} \hat{\rho}} \\
& +\frac{1}{288}\left(-\frac{1}{2}\left(\left(e_{0}\right)_{a}^{\hat{\mu}} \Gamma_{\hat{\mu}} H_{1}+\left(e_{1}\right)_{a}^{\hat{\mu}} \Gamma_{\hat{\mu}} H_{0}\right)+\frac{3}{2}\left(H_{0}\left(e_{1}\right)_{a}^{\hat{\mu}} \Gamma_{\hat{\mu}}+H_{1}\left(e_{0}\right)_{a}^{\hat{\mu}} \Gamma_{\hat{\mu}}\right)\right), \\
& \Pi_{2}^{(a)}=\frac{1}{4}\left(\omega_{a}^{\hat{\nu} \hat{\rho}}\right)_{0} \Gamma_{\hat{\nu} \hat{\rho}}+\frac{1}{288}\left(-\frac{1}{2}\left(e_{0}\right)_{a}^{\hat{\mu}} \Gamma_{\hat{\mu}} H_{0}+\frac{3}{2} \not H_{0}\left(e_{0}\right)_{a}^{\hat{\mu}} \Gamma_{\hat{\mu}}\right), \\
& \Pi_{3}=\frac{1}{4}\left(\omega_{r}^{\hat{\nu} \hat{\rho}}\right)_{1} \Gamma_{\hat{\nu} \hat{\rho}} \\
& +\frac{1}{288}\left(-\frac{1}{2}\left(\left(e_{0}\right)_{r}^{\hat{\mu}} \Gamma_{\hat{\mu}} H_{1}+\left(e_{1}\right)_{r}^{\hat{\mu}} \Gamma_{\hat{\mu}} F_{0}\right)+\frac{3}{2}\left(F_{0}\left(e_{1}\right)_{r}^{\hat{\mu}} \Gamma_{\hat{\mu}}+F_{1}\left(e_{0}\right)_{r}^{\hat{\mu}} \Gamma_{\hat{\mu}}\right)\right) \text {, } \\
& \Pi_{4}=\frac{1}{4}\left(\omega_{r}^{\hat{\nu} \hat{\rho}}\right)_{0} \Gamma_{\hat{\nu} \hat{\rho}}+\frac{1}{288}\left(-\frac{1}{2}\left(e_{0}\right)_{r}^{\hat{\mu}} \Gamma_{\hat{\mu}} F_{0}+\frac{3}{2} F_{0}\left(e_{0}\right)_{r}^{\hat{\mu}} \Gamma_{\hat{\mu}}\right), \\
& \Pi_{5}^{(m)}=e^{\frac{R(0)}{6}}\left(-h_{\Omega}\left(e_{0}\right)_{m}^{\hat{m}}+\frac{1}{6} \sigma^{c} \partial_{c} R\left(e_{0}\right)_{m}^{\hat{m}}+\left(e_{1}\right)_{m}^{\hat{m}}\right) \Gamma_{\Omega} \Gamma_{\hat{m}}, \\
& \Pi_{6}^{(m)}=e^{\frac{R(0)}{6}}\left(e_{0}\right)_{m}^{\hat{m}} \Gamma_{\Omega} \Gamma_{\hat{m}}, \\
& \Pi_{7}^{(m)}=\frac{1}{6} \partial^{r} R(0)\left(e_{0}\right)_{r}^{\hat{r}}\left(e_{1}\right)_{m}^{\hat{m}} \Gamma_{\hat{r}} \Gamma_{\hat{m}} \\
& +\frac{1}{6}\left(\partial^{r} R(0)\left(e_{1}\right)_{r}^{\hat{\mu}}+\sigma^{c} \partial_{c} \partial^{r} R\left(e_{0}\right)_{r}^{\hat{\mu}}+\partial^{a} R\left(e_{0}\right)_{a}^{\hat{\mu}}\right) \Gamma_{\hat{\mu}} \Gamma_{\hat{m}}\left(e_{0}\right)_{m}^{\hat{m}} \\
& -\frac{1}{2} \partial^{r} h_{\Omega}\left(e_{0}\right)_{r}^{\hat{r}}\left(e_{0}\right)_{m}^{\hat{m}} \Gamma_{\hat{r}} \Gamma_{\hat{m}} \\
& +\frac{1}{288}\left(-\left(e_{0}\right)_{m}^{\hat{m}} \Gamma_{\hat{m}} H_{1}-\left(e_{1}\right)_{m}^{\hat{m}} \Gamma_{\hat{m}} H_{0}+3\left(F_{0}\left(e_{1}\right)_{m}^{\hat{m}} \Gamma_{\hat{m}}+F_{1}\left(e_{0}\right)_{m}^{\hat{m}} \Gamma_{\hat{m}}\right)\right), \\
& \Pi_{8}^{(m)}=\frac{1}{6} \partial^{r} R(0)\left(e_{0}\right)_{r}^{\hat{r}}\left(e_{0}\right)_{m}^{\hat{m}} \Gamma_{\hat{r}} \Gamma_{\hat{m}}+\frac{1}{288}\left(-\left(e_{0}\right)_{m}^{\hat{m}} \Gamma_{\hat{m}} F_{0}+3 F_{0}\left(e_{0}\right)_{m}^{\hat{m}} \Gamma_{\hat{m}}\right) \text {. }
\end{aligned}
$$

By further implementing the ansatz (5.11) for $\eta_{1}$ and collecting the $\sigma$-linear part of the operators $\Pi_{o d d}$,

$$
\Pi_{o d d}\left(\sigma, r, y^{i}\right)=\bar{\Pi}_{o d d}\left(r, y^{i}\right)+\sigma^{a} \Pi_{o d d, a}\left(r, y^{i}\right)
$$

we find trivially that the equations (A.15)-(A.17) split into the two independent groups (5.12)-(5.14) and (5.15)-(5.17). In section 5.1 we focused on the $\sigma$-linear part of the Killing spinor equations (A.15).

\section{A.2 Extrinsic perturbations}

The analysis of the extrinsic perturbations proceeds in a similar fashion. The metric deformation is now given by eq. (6.2). It is convenient to choose a gauge where

$$
h_{\mu \nu}(r, \phi) d x^{\mu} d x^{\nu}=\cos \phi\left(\tilde{h}_{a b}(r) d \sigma^{a} d \sigma^{b}+e^{-\frac{R}{3}}\left(\tilde{h}_{r}(r) \frac{d r^{2}}{r^{2}}+\tilde{h}_{\Omega}(r)\left(d \phi^{2}+\sin ^{2} \phi d \Omega_{3}^{2}\right)\right)\right) .
$$

For further details about this choice we refer the reader to [33] and references therein. The four-form flux $F_{4}$ and the Killing spinor $\eta$ are perturbed as in (6.3), (6.6). 
A useful fact about covariant derivatives of spinors along the $S^{3}$ directions is that they can be written in terms of covariant derivatives on the unit $S^{3}$ as follows

$$
\nabla_{m} \eta=\nabla_{m}^{\left(S^{3}\right)} \eta-\frac{1}{4} \partial^{\nu}\left(\log \left(e^{-\frac{R}{3}} \sin ^{2} \phi\left(1+\delta \tilde{h}_{\Omega} \cos \phi\right)\right)\right) \Gamma_{\nu m} \eta
$$

Here $m$ is an $S^{3}$ index. Since $\eta_{1}$ is an $S^{3}$ Killing spinor (6.7) we find

$$
\boldsymbol{\delta} \nabla_{m} \eta_{1}=\frac{i \tilde{C}}{2 \sin \phi} e^{\frac{R}{6}} \Gamma_{m} \boldsymbol{\delta} \eta_{1}+\frac{1}{2} e^{\frac{R}{3}}\left(\frac{1}{6} r^{2} \partial_{r} R \Gamma_{r m}-\Gamma_{\phi m}\right) \boldsymbol{\delta} \eta_{1}+\mathcal{O}\left(\boldsymbol{\delta}^{2}\right)
$$

The covariant derivative of $\eta_{0}$, which is an $S^{4}$ Killing spinor, can be deduced using the relation ${ }^{9}$

$$
\nabla_{m}^{\left(S^{4}\right)} \eta_{0}=\nabla_{m}^{\left(S^{3}\right)} \eta_{0}-\frac{1}{2} \cot \phi \Gamma_{\phi m}^{\left(S^{4}\right)} \eta_{0}
$$

Together with the defining relation of $S^{4}$ Killing spinors we obtain

$$
\nabla_{m}^{\left(S^{3}\right)} \eta_{0}=\frac{1}{2} e^{\frac{R}{6}}\left(1-\frac{1}{2} \boldsymbol{\delta} \tilde{h}_{\Omega}\right)\left(C \Gamma_{\Omega} \Gamma_{m}-\cot \phi \Gamma_{\hat{\phi}} \Gamma_{m}\right) \eta_{0}
$$

Repeating the steps of the previous subsection A.1 (appropriately adapted) we find that the Killing spinor equations (A.14) take the form

$$
\begin{aligned}
\Sigma_{1}^{(a)} \eta_{0}+\Sigma_{2}^{(a)} \eta_{1} & =0, \\
\partial_{r} \eta_{1}+\Sigma_{3} \eta_{0}+\Sigma_{4} \eta_{1} & =0, \\
\partial_{\phi} \eta_{1}+\Sigma_{5} \eta_{0}+\Sigma_{6} \eta_{1} & =0, \\
\left(C \Sigma_{7}^{(m)}+\Sigma_{9}^{(m)}\right) \eta_{0}+\left(\tilde{C} \Sigma_{8}^{(m)}+\Sigma_{10}^{(m)}\right) \eta_{1} & =0 .
\end{aligned}
$$

$m$ is again an $S^{3}$ index. The general expression of the operators $\Sigma_{1}^{(a)}, \Sigma_{2}^{(a)}, \Sigma_{3}, \Sigma_{4}$ is the

${ }^{9} \Gamma_{\mu}^{\left(S^{4}\right)}$ denotes a curved index $\Gamma$-matrix in $S^{4}$. 
same as in (A.18)-(A.21). The remaining operators are

$$
\begin{aligned}
\Sigma_{5}= & \frac{1}{4}\left(\omega_{\phi}^{\hat{\nu} \hat{\rho}}\right)_{1} \Gamma_{\hat{\nu} \hat{\rho}}+\frac{1}{288}\left(-\frac{1}{2}\left(\left(e_{0}\right)_{\phi}^{\hat{\phi}} \Gamma_{\hat{\phi}} H_{1}+\left(e_{1}\right)_{\phi}^{\hat{\phi}} \Gamma_{\hat{\phi}} H_{0}\right)\right. \\
& \left.+\frac{3}{2}\left(H_{0}\left(e_{1}\right)_{\phi}^{\hat{\phi}} \Gamma_{\hat{\phi}}+H_{1}\left(e_{0}\right)_{\phi}^{\hat{\phi}} \Gamma_{\hat{\phi}}\right)\right), \\
\Sigma_{6}= & \frac{1}{4}\left(\omega_{\phi}^{\hat{\nu} \hat{\rho}}\right)_{0} \Gamma_{\hat{\nu} \hat{\rho}}+\frac{1}{288}\left(-\frac{1}{2}\left(e_{0}\right)_{\phi}^{\hat{\phi}} \Gamma_{\hat{\phi}} H_{0}+\frac{3}{2} H_{0}\left(e_{0}\right)_{\phi}^{\hat{\phi}} \Gamma_{\hat{\phi}}\right), \\
\Sigma_{7}^{(m)}= & \frac{1}{2} e^{\frac{R}{6}} \Gamma_{\Omega} \Gamma_{\hat{m}}\left(\left(e_{1}\right)_{m}^{\hat{m}}-\frac{1}{2} \tilde{h}_{\Omega}\left(e_{0}\right)_{m}^{\hat{m}}\right) \\
\Sigma_{8}^{(m)}= & \frac{i}{2 \sin \phi} e^{\frac{R}{6}} \Gamma_{\hat{m}}\left(e_{0}\right)_{m}^{\hat{m}}, \\
\Sigma_{9}^{(m)}= & -\frac{1}{2} e^{\frac{R}{6}} \cot \phi \Gamma_{\hat{\phi}} \Gamma_{\hat{m}}\left(\left(e_{1}\right)_{m}^{\hat{m}}-\frac{1}{2} \tilde{h}_{\Omega}\left(e_{0}\right)_{m}^{\hat{m}}\right) \\
& -\frac{1}{4} \partial_{\nu} \log \left(e^{-\frac{R}{3}} \sin ^{2} \phi\right)\left(\left(e_{1}\right)_{\hat{\nu}}^{\nu}\left(e_{0}\right)_{m}^{\hat{m}}+\left(e_{0}\right)_{\hat{\nu}}^{\nu}\left(e_{1}\right)_{m}^{\hat{m}}\right) \Gamma^{\hat{\nu}} \Gamma_{\hat{m}} \\
& -\frac{1}{4} \partial_{\nu}\left(\tilde{h}_{\Omega} \cos \phi\right)\left(e_{0}\right)_{\hat{\nu}}^{\nu}\left(e_{0}\right)_{m}^{\hat{m}} \Gamma^{\hat{\nu}} \Gamma_{\hat{\mu}} \\
& +\frac{1}{288}\left(-\frac{1}{2}\left(\left(e_{0}\right)_{m}^{\hat{m}} \Gamma_{\hat{m}} H_{1}+\left(e_{1}\right)_{m}^{\hat{m}} \Gamma_{\hat{m}} H_{0}\right)+\frac{3}{2}\left(H_{0}\left(e_{1}\right)_{m}^{\hat{m}} \Gamma_{\hat{m}}+H_{1}\left(e_{0}\right)_{m}^{\hat{m}} \Gamma_{\hat{m}}\right)\right), \\
\Sigma_{10}^{(m)}= & \frac{1}{2} e^{\frac{R}{6}}\left(\frac{1}{6} r \partial_{r} R \Gamma_{\hat{r}}-\Gamma_{\hat{\phi}}\right) \Gamma_{\hat{m}}\left(e_{0}\right)_{m}^{\hat{m}}+\frac{1}{288}\left(-\frac{1}{2}\left(e_{0}\right)_{m}^{\hat{m}} \Gamma_{\hat{m}} H_{0}+\frac{3}{2} F_{0}\left(e_{0}\right)_{m}^{\hat{m}} \Gamma_{\hat{m}}\right)
\end{aligned}
$$

Implementing the ansatz (6.6) and collecting separately the pieces that depend linearly on $r K_{a b}$ we find that eqs. (A.32)-(A.35) split into two independent groups. In section 6.1 we focused on a specific part, (6.12), of the $\left(r K_{a b}\right)$-linear group.

\section{B Useful identities}

\section{B.1 Consistency check of (5.23)}

The pertubative Killing spinor equations (5.23) imply

$$
\Pi_{2} \partial_{a} \eta=-\Pi_{1, a} \eta_{0} \Rightarrow\left(\frac{1}{2 r_{H}^{3}} \Pi_{2}-1\right) \Pi_{1, a} \eta_{0}=0
$$

where we multiplied simultaneously both sides by $\Pi_{2}$, used the identity

$$
\Pi_{2}^{2}=2 r_{H}^{3} \Pi_{2},
$$

and re-applied the equation (5.23). In this appendix we examine the validity of the consistency equation (B.1).

For convenience let us write

$$
\Pi_{1, a}=Q_{a}+R_{a}
$$

with

$$
\begin{aligned}
& Q_{a}=\partial_{a} r_{H}^{3}+\partial_{a}\left(\sin \theta r_{H}^{3}\right) \Gamma_{\|}+\partial_{a}\left(\cos \theta r_{H}^{3}\right) \Gamma_{\|} \Gamma_{\perp}, \\
& R_{a}=-(1+C \cos \theta) r_{H}^{3}(0) \sum_{c_{\perp}}\left(-\partial_{a} u_{c_{\perp}} \Gamma^{\hat{0} \hat{c}_{\perp}}+\partial_{a} v_{c_{\perp}} \Gamma^{\hat{1} \hat{c}_{\perp}}+\partial_{a} w_{c_{\perp}} \Gamma^{\hat{2} \hat{c}_{\perp}}\right) .
\end{aligned}
$$


With a bit of algebra one can show that

$$
\left(\frac{1}{2 r_{H}^{3}} \Pi_{2}-1\right) Q_{a} \eta_{0}=\frac{C \sin \theta \partial_{a} \theta}{2(1+C \cos \theta)} \Pi_{2} \eta_{0}=0
$$

and

$$
\left(\frac{1}{2 r_{H}^{3}} \Pi_{2}-1\right) R_{a} \eta_{0}=\frac{1}{2} \sin \theta \sum_{c_{\perp}}\left(-\partial_{b} u_{c_{\perp}} \Gamma^{\hat{0} \hat{c}_{\perp}}+\partial_{b} v_{c_{\perp}} \Gamma^{\hat{1} \hat{c}_{\perp}}+\partial_{b} w_{c_{\perp}} \Gamma^{\hat{2} \hat{c}_{\perp}}\right) \Gamma_{\|} \Pi_{2} \eta_{0}=0 .
$$

We conclude that the consistency equation (B.1) is satisfied automatically as it should.

\section{B.2 An identity for Killing spinors on $S^{4}$}

In this appendix we prove the identity (6.17) that was employed in section 6.1. Following section 3.3 we consider a spinor $\eta$ in eleven dimensions whose four-sphere part is a Killing spinor on the unit $S^{4}$. By definition, the covariant derivatives of $\eta$ on $S^{4}$ obeys the identity

$$
\nabla_{j} \eta=\frac{C}{2} \Gamma_{\Omega} \Gamma_{j} \eta, \quad C= \pm 1
$$

$\Gamma_{\Omega}$ is the chirality operator on $S^{4}$ and $\Gamma_{j}=e_{j}^{\hat{j}} \Gamma_{\hat{j}}$ with $e_{j}^{\hat{j}}$ the vielbein on the unit $S^{4}$. In addition, we require the identity (3.30), equivalently

$$
\Gamma_{\hat{r}} \Gamma_{\Omega} \eta=C \eta
$$

In hyperspherical coordinates, where the metric of the unit four-sphere is

$$
d \Omega_{4}^{2}=d \phi^{2}+\sin ^{2} \phi d \Omega_{3}^{2},
$$

$\eta$ takes the form [48]

$$
\eta=e^{\frac{C}{2} \phi \Gamma_{\Omega} \Gamma_{\hat{\phi}} \tilde{\eta}}, \text { such that } \partial_{\phi} \tilde{\eta}=0
$$

A convenient standard identity of $\Gamma$-matrix exponentials reads

$$
e^{\frac{C}{2} \phi \Gamma_{\Omega} \Gamma_{\hat{\phi}}}=\cos \left(\frac{\phi}{2}\right) \cdot \mathbf{1}+C \sin \left(\frac{\phi}{2}\right) \Gamma_{\Omega} \Gamma_{\hat{\phi}} \cdot
$$

Combining (B.9) with the more explicit form (B.11) it is straightforward to show that

$$
\left[\cos \left(\frac{\phi}{2}\right) \Gamma_{\hat{r}}-\sin \left(\frac{\phi}{2}\right) \Gamma_{\hat{\phi}}\right] \eta=0
$$

Applying this equation at angle $2 \phi$ and using $\eta(2 \phi)=e^{\frac{C}{2} \phi \Gamma_{\Omega} \Gamma_{\hat{\phi}}} \eta(\phi)$ we arrive easily at the required identity $(6.17)$

$$
\left(\cos \phi \Gamma_{\hat{r}}-\sin \phi \Gamma_{\hat{\phi}}\right) \eta=0 .
$$

Open Access. This article is distributed under the terms of the Creative Commons Attribution License (CC-BY 4.0), which permits any use, distribution and reproduction in any medium, provided the original author(s) and source are credited. 


\section{References}

[1] A.A. Tseytlin, Born-Infeld action, supersymmetry and string theory, hep-th/9908105 [INSPIRE].

[2] R.C. Myers, NonAbelian phenomena on D-branes, Class. Quant. Grav. 20 (2003) S347 [hep-th/0303072] [INSPIRE].

[3] J. Simon, Brane Effective Actions, א-symmetry and Applications, Living Rev. Rel. 15 (2012) 3 [arXiv: 1110.2422] [INSPIRE].

[4] R. Emparan, D. Mateos and P.K. Townsend, Supergravity supertubes, JHEP 07 (2001) 011 [hep-th/0106012] [INSPIRE].

[5] O. Lunin, Strings ending on branes from supergravity, JHEP 09 (2007) 093 [arXiv:0706.3396] [INSPIRE].

[6] O. Lunin, Brane webs and 1/4-BPS geometries, JHEP 09 (2008) 028 [arXiv:0802.0735] [INSPIRE].

[7] L. Martucci, Electrified branes, JHEP 02 (2012) 097 [arXiv:1110.0627] [INSPIRE].

[8] J.P. Gauntlett, Branes, calibrations and supergravity, hep-th/0305074 [INSPIRE].

[9] R. Emparan, T. Harmark, V. Niarchos and N.A. Obers, World-Volume Effective Theory for Higher-Dimensional Black Holes, Phys. Rev. Lett. 102 (2009) 191301 [arXiv:0902.0427] [INSPIRE].

[10] R. Emparan, T. Harmark, V. Niarchos and N.A. Obers, Essentials of Blackfold Dynamics, JHEP 03 (2010) 063 [arXiv:0910.1601] [INSPIRE].

[11] D. Gorbonos and B. Kol, A Dialogue of multipoles: Matched asymptotic expansion for caged black holes, JHEP 06 (2004) 053 [hep-th/0406002] [INSPIRE].

[12] S. Bhattacharyya, V.E. Hubeny, S. Minwalla and M. Rangamani, Nonlinear Fluid Dynamics from Gravity, JHEP 02 (2008) 045 [arXiv:0712.2456] [INSPIRE].

[13] R. Emparan, T. Harmark, V. Niarchos, N.A. Obers and M.J. Rodriguez, The Phase Structure of Higher-Dimensional Black Rings and Black Holes, JHEP 10 (2007) 110 [arXiv:0708.2181] [INSPIRE].

[14] J. Camps, R. Emparan, P. Figueras, S. Giusto and A. Saxena, Black Rings in Taub-NUT and D0-D6 interactions, JHEP 02 (2009) 021 [arXiv:0811.2088] [INSPIRE].

[15] R. Emparan, T. Harmark, V. Niarchos and N.A. Obers, New Horizons for Black Holes and Branes, JHEP 04 (2010) 046 [arXiv:0912.2352] [INSPIRE].

[16] J. Armas and N.A. Obers, Blackfolds in (Anti)-de Sitter Backgrounds, Phys. Rev. D 83 (2011) 084039 [arXiv: 1012.5081] [INSPIRE].

[17] M.M. Caldarelli, R. Emparan and B. Van Pol, Higher-dimensional Rotating Charged Black Holes, JHEP 04 (2011) 013 [arXiv:1012.4517] [INSPIRE].

[18] R. Emparan, T. Harmark, V. Niarchos and N.A. Obers, Blackfolds in Supergravity and String Theory, JHEP 08 (2011) 154 [arXiv:1106.4428] [INSPIRE].

[19] G. Grignani, T. Harmark, A. Marini, N.A. Obers and M. Orselli, Thermal string probes in AdS and finite temperature Wilson loops, JHEP 06 (2012) 144 [arXiv:1201.4862] [INSPIRE].

[20] J. Armas, T. Harmark, N.A. Obers, M. Orselli and A.V. Pedersen, Thermal Giant Gravitons, JHEP 11 (2012) 123 [arXiv: 1207.2789] [INSPIRE]. 
[21] G. Grignani, T. Harmark, A. Marini, N.A. Obers and M. Orselli, Heating up the BIon, JHEP 06 (2011) 058 [arXiv: 1012.1494] [INSPIRE].

[22] G. Grignani, T. Harmark, A. Marini, N.A. Obers and M. Orselli, Thermodynamics of the hot BIon, Nucl. Phys. B 851 (2011) 462 [arXiv:1101.1297] [InSPIRE].

[23] V. Niarchos and K. Siampos, M2-M5 blackfold funnels, JHEP 06 (2012) 175 [arXiv: 1205.1535] [INSPIRE].

[24] V. Niarchos and K. Siampos, Entropy of the self-dual string soliton, JHEP 07 (2012) 134 [arXiv: 1206.2935] [INSPIRE].

[25] V. Niarchos and K. Siampos, The black M2-M5 ring intersection spins, PoS (Corfu2012) 088 [arXiv: 1302.0854] [INSPIRE].

[26] J. Armas, N.A. Obers and A.V. Pedersen, Null-Wave Giant Gravitons from Thermal Spinning Brane Probes, JHEP 10 (2013) 109 [arXiv:1306.2633] [INSPIRE].

[27] J. Armas, J. Gath and N.A. Obers, Electroelasticity of Charged Black Branes, JHEP 10 (2013) 035 [arXiv: 1307.0504] [INSPIRE].

[28] G. Grignani, T. Harmark, A. Marini and M. Orselli, Thermal DBI action for the D3-brane at weak and strong coupling, JHEP 03 (2014) 114 [arXiv:1311.3834] [INSPIRE].

[29] R. Emparan, V.E. Hubeny and M. Rangamani, Effective hydrodynamics of black D3-branes, JHEP 06 (2013) 035 [arXiv: 1303.3563] [INSPIRE].

[30] M.M. Caldarelli, J. Camps, B. Goutéraux and K. Skenderis, AdS/Ricci-flat correspondence and the Gregory-Laflamme instability, Phys. Rev. D 87 (2013) 061502 [arXiv:1211.2815] [INSPIRE].

[31] M.M. Caldarelli, J. Camps, B. Goutéraux and K. Skenderis, AdS/Ricci-flat correspondence, JHEP 04 (2014) 071 [arXiv: 1312.7874] [INSPIRE].

[32] J. Camps, R. Emparan and N. Haddad, Black Brane Viscosity and the Gregory-Laflamme Instability, JHEP 05 (2010) 042 [arXiv:1003.3636] [INSPIRE].

[33] J. Camps and R. Emparan, Derivation of the blackfold effective theory, JHEP 03 (2012) 038 [Erratum ibid. 1206 (2012) 155] [arXiv:1201.3506] [INSPIRE].

[34] J. Gath and A.V. Pedersen, Viscous asymptotically flat Reissner-Nordström black branes, JHEP 03 (2014) 059 [arXiv: 1302.5480] [INSPIRE].

[35] C.G. Callan Jr., J.A. Harvey and A. Strominger, Worldbrane actions for string solitons, Nucl. Phys. B 367 (1991) 60 [InSPIRE].

[36] G.W. Gibbons and P.K. Townsend, Vacuum interpolation in supergravity via super p-branes, Phys. Rev. Lett. 71 (1993) 3754 [hep-th/9307049] [INSPIRE].

[37] P. Pasti, D.P. Sorokin and M. Tonin, On Lorentz invariant actions for chiral p forms, Phys. Rev. D 55 (1997) 6292 [hep-th/9611100] [INSPIRE].

[38] P. Pasti, D.P. Sorokin and M. Tonin, Covariant action for a $D=11$ five-brane with the chiral field, Phys. Lett. B 398 (1997) 41 [hep-th/9701037] [INSPIRE].

[39] I.A. Bandos, K. Lechner, A. Nurmagambetov, P. Pasti, D.P. Sorokin et al., Covariant action for the superfive-brane of M-theory, Phys. Rev. Lett. 78 (1997) 4332 [hep-th/9701149] [INSPIRE]. 
[40] S.-L. Ko, D. Sorokin and P. Vanichchapongjaroen, The M5-brane action revisited, JHEP 11 (2013) 072 [arXiv: 1308.2231] [INSPIRE].

[41] M. Perry and J.H. Schwarz, Interacting chiral gauge fields in six-dimensions and Born-Infeld theory, Nucl. Phys. B 489 (1997) 47 [hep-th/9611065] [INSPIRE].

[42] E. Bergshoeff, D.S. Berman, J.P. van der Schaar and P. Sundell, A Noncommutative M-theory five-brane, Nucl. Phys. B 590 (2000) 173 [hep-th/0005026] [INSPIRE].

[43] R. Gopakumar, S. Minwalla, N. Seiberg and A. Strominger, (OM) theory in diverse dimensions, JHEP 08 (2000) 008 [hep-th/0006062] [INSPIRE].

[44] J.P. Gauntlett and S. Pakis, The Geometry of D = 11 Killing spinors, JHEP 04 (2003) 039 [hep-th/0212008] [INSPIRE].

[45] J.M. Izquierdo, N.D. Lambert, G. Papadopoulos and P.K. Townsend, Dyonic membranes, Nucl. Phys. B 460 (1996) 560 [hep-th/9508177] [InSPIRE].

[46] T. Harmark, Open branes in space-time noncommutative little string theory, Nucl. Phys. B 593 (2001) 76 [hep-th/0007147] [INSPIRE].

[47] T. Harmark and N.A. Obers, Phase structure of noncommutative field theories and spinning brane bound states, JHEP 03 (2000) 024 [hep-th/9911169] [INSPIRE].

[48] H. Lü, C.N. Pope and J. Rahmfeld, A Construction of Killing spinors on $S^{n}$, J. Math. Phys. 40 (1999) 4518 [hep-th/9805151] [INSPIRE].

[49] J. Armas, J. Gath, V. Niarchos, N.A. Obers and A.V. Pedersen, to appear. 